\title{
Value-directed memory effects on item and context memory
}

\author{
Jonathan J. Villaseñor ${ }^{1}$ - Allison M. Sklenar ${ }^{1}$. Andrea N. Frankenstein ${ }^{1} \cdot$ Pauline Urban Levy $^{1} \cdot$ Matthew P. McCurdy $^{1}$. \\ Eric D. Leshikar ${ }^{1}$
}

Accepted: 4 February 2021 / Published online: 26 February 2021

(C) The Psychonomic Society, Inc. 2021

\begin{abstract}
The ability to prioritize learning some information over others when that information is considered important or valuable is known as value-directed remembering. In these experiments, we investigate how value influences different aspects of memory, including item memory (memory for the to-be-learned materials) and context memory (memory for peripheral details that occurred when studying items) to get a better understanding of how people prioritize learning information. In this investigation, participants encoded words associated with a range of values (binned into higher, medium, and lower value in Experiment 1, and into higher and lower value in Experiment 2) for a subsequent memory test that measured item memory (Is this item old or new?) as well as both objective context memory (memory for an objectively verifiable contextual detail: In which voice was this item spoken?) and subjective context memory (How many visual, auditory, and extraneous thoughts/feelings can you remember associated with this item?). Results indicated that value influenced item memory but had no effect on objective context memory in both Experiments. In Experiment 2, results showed better subjective context memory for multiple episodic details for highervalue relative to lower-value materials. Overall, these findings suggest that value has a strong influence over some aspects of memory, but not others. This work gives a richer understanding of how people prioritize learning more important over less important information.
\end{abstract}

Keywords Value-directed remembering $\cdot$ Item memory $\cdot$ Source memory $\cdot$ Context memory $\cdot$ Memory characteristics questionnaire

Evidence suggests that people are able to prioritize learning some information over others when that information is considered important or valuable (Castel, 2007; Castel, Benjamin, Craik, \& Watkins, 2002; Castel, Farb, \& Craik, 2007). One method to study prioritization of information in memory is a procedure called value-directed remembering (VDR). In a typical VDR task, participants are asked to study items associated with varying degrees of value (e.g., items associated with values ranging from 1 to 8 ) before then completing a memory test. Instructions in VDR tasks often direct participants to remember as many studied items as possible, but also to prioritize learning higher-value (e.g., a value of 8 ) relative to lower-value materials (e.g., value of 1). Results from VDR experiments typically show that participants are better able to remember higher-value relative to lower-value materials,

Eric D. Leshikar

leshikar@uic.edu

1 Department of Psychology, University of Illinois at Chicago, $1007 \mathrm{~W}$ Harrison St (M/C 285), Chicago, IL 60607, USA reflecting the ability to prioritize information in memory (Castel, 2007; Castel et al., 2011; Middlebrooks, Kerr, \& Castel, 2017; Robison \& Unsworth, 2017; Spaniol, Schain, \& Bowen, 2014). Remembering valuable materials has been found across various types of stimuli, such as words (Castel et al., 2002), pictures (Castel, 2005; , \& Reber, 2019), grocery prices (Castel, 2005), faces (DeLozier \& Rhodes, 2015; Hargis \& Castel, 2017), names (Hargis \& Castel, 2017), and occupations (Hargis \& Castel, 2017), suggesting that people can readily remember valuable information. In the present study, we extend knowledge on VDR by investigating the influence of value on both item memory (i.e., studied words) and context memory (peripheral episodic details associated with studied words).

The majority of work on VDR has predominantly investigated the ability to remember higher-value relative to lowervalue materials for item memory (Castel, 2005; Castel et al., 2002; Castel et al., 2007; Castel, Murayama, Friedman, McGillivray, \& Link, 2013; Hargis \& Castel, 2017; Hayes, Kelly, \& Smith, 2013; Middlebrooks et al., 2017; Middlebrooks, Murayama, \& Castel, 2016). One common 
experimental approach in past VDR work is to assign value to materials either immediately before or concurrent with (i.e., at the same time as) presentation of to-be-learned information (Castel, 2005; Castel et al., 2013; Cohen et al., 2019; Cohen, Rissman, Suthana, Castel, \& Knowlton, 2016; Middlebrooks et al., 2016). Although this procedure (presenting value before or concurrent with presentation of studied items) has been crucial in understanding how value influences memory, less is known about how participants prioritize information when the value of to-be-learned material is presented after the stimulus is encountered. In the real world, the importance (or value) of information is sometimes not evident until after exposure to information (e.g., a student hearing that a concept will be on an upcoming exam). In one of the few studies that has examined VDR effects when value is presented after the stimuli, participants studied words associated with varying values, followed by both recall and recognition memory tests (Hayes et al., 2013). Results indicated better memory for higher-value relative to lower-value words as measured by both recall and recognition, which is consistent with findings from studies where value is presented before (or concurrent with) to-belearned materials (Castel et al., 2007; Cohen, Rissman, Hovhannisyan, Castel, \& Knowlton, 2017). These findings in Hayes et al. (2013) may suggest that processes used to prioritize learning valuable information may be similar whether value is known before or after stimuli are presented. Given the limited work in this domain, direct comparison between conditions where value is known before or after an episode takes place will advance understanding on how participants prioritize learning valuable information. Specifically, finding a pattern of results where item and context memory differ dependent on when value is known could imply that encoding processes might be different in value-before versus value-after conditions. In this experiment, we directly compare the influence of value on memory dependent on whether value is known before or after materials are presented.

Although the majority of VDR work has focused on item memory, less work has examined the extent that value influences context memory. Context memory can be described as memory for extraneous episodic details encountered at the time of study (James, Rajah, \& Duarte, 2019; Naveh-Benjamin \& Craik, 1995; Rugg et al., 2012; Spencer \& Raz, 1995), which can include perceptual details (color details of visual stimuli; acoustic details of auditory stimuli), source information (whether materials were presented in one list versus another, etc.), or even self-generated details (extraneous thoughts or feelings induced by studied material). Even further, context memory can be categorized into objective context (memory for objectively verifiable details, such as source information) or subjective context (memory for details that are not objectively verifiable, such as the "amount" of details one can retrieve about perceptual or self-generated thoughts/feelings). Given that people show item memory advantages for higher-value compared with lower-value materials, it may be that there is a similar pattern for both objective and subjective context memory: When people prioritize learning some content over others, they encode a detail-rich episodic representation that includes numerous contextual details. Looking at findings from objective context memory first, results of several VDR experiments show better objective context memory (e.g., location in which a studied word appeared) for higher-value compared with lower-value items, suggesting that at least some contextual details (location) are enhanced for valuable information (Cohen et al., 2019; Elliott, McClure, \& Brewer, 2020; Siegel \& Castel, 2018a, Experiments 1 \& 2; Shigemune, Tsukiura, Kambara, \& Kawashima, 2014; Schwartz, Siegel, \& Castel, 2020). Interestingly, though, not all investigations have shown item and objective context memory enhancements for highervalue relative to lower-value materials. In one study, older (and younger) participants encoded words presented in different font colors (red, yellow, lime green, or cyan blue; Hennessee, Knowlton, \& Castel, 2018, Experiment 1). Results showed that older adults exhibited item memory increases and concomitant context memory (font color) decreases for higher-value relative to lower-value materials. This finding within the older adults suggests possible item-context trade-offs in memory (Jurica \& Shimamura, 1999), at least under certain situations, such as in aging populations. Because some work suggests that value effects on memory are contingent on controlled use of cognitive processes (i.e., top-down processes) at encoding (Castel, Balota, \& McCabe, 2009; Castel, Lee, Humphreys, \& Moore, 2011; Robison \& Unsworth, 2017), this may mean that allocation of resources to encode materials might yield trade-offs in memory with enhanced memory for some details (i.e., item memory) that come at the expense of reduced memory for other details (i.e., context memory). This could partially account for studies that have shown no context memory benefit for highervalue compared with lower-value items in the past (Castel, 2005; Castel et al., 2007; Hennessee, Castel, \& Knowlton, 2017; Hennessee et al., 2018; Hennessee, Patterson, Castel, \& Knowlton, 2019; Stefanidi, Ellis, \& Brewer, 2018). Further still, it may be that item-context trade-offs, if they exist, are exacerbated under some conditions, such as when value is not known until after stimuli have been studied. Specifically, it may be that item and objective context memory are both enhanced when value is known before a trial since participants may allocate resources in a top-down fashion to encode various episodic details associated with items. In contrast, this allocation of resources may not be possible when value is known after. Given the mixed objective context memory findings in past work (Castel, 2005; Castel et al., 2007; Hennessee et al., 2017; Hennessee et al., 2018; Hennessee et al., 2019; Stefanidi et al., 2018), we investigate the extent context memory as measured by voice source (In which voice was this word spoken?) is influenced by value depending on whether value is presented before or after the learning episode. 
Turning to subjective context memory, there is some evidence that participants subjectively report more contextual details associated with higher-value compared with lowervalue materials. For example, some VDR experiments have used remember/know judgments at retrieval to evaluate subjective context memory. In these studies, participants make subjective "remember" judgments if they retrieve any contextual details associated with trials; however, these remember decisions are considered subjective because the exact details participants retrieve to make such remember judgments are not directly measured. Results from past VDR experiments using remember/know procedures have consistently shown that rates of "remember" responses are enhanced for highervalued relative to lower-valued materials (Cohen et al., 2017; Elliott \& Brewer, 2019; Elliott et al., 2020; Hennessee et al., 2017). Because the type of contextual details participants retrieves to make remember judgments is unclear, it is unknown what contextual details are enhanced for higher-valued compared with lower-valued materials. A richer way to evaluate memory for subjective contextual details is through use of a memory characteristic questionnaire (MCQ; Johnson, Foley, Suengas, \& Raye, 1988). In MCQ tasks, participants give a subjective rating for the "amount" of contextual details they can retrieve for a range of different details, such as visual information, auditory information, and extraneous (selfgenerated) thoughts and feelings. MCQs are useful because they allow a more precise way to assess the contents of memory than using remember/know procedures. Even further, results from MCQ assessments can be used in combination with objective memory measures (such as source) to gain a more complete understanding of memory phenomenon. For instance, one study used MCQs to assess context memory for words processed in reference to the self (Leshikar, Dulas, \& Duarte, 2015). Although not a VDR experiment, results from Leshikar et al. (2015) showed that self-referentially processing information resulted in higher rates of both objective (source memory) and subjective (MCQ) measures of context memory, which led to a more nuanced understanding of self-referential memory effects. In the present experiment, we assess both objective (source) and subjective context memory (MCQ) to pursue more precise understanding of how value influences context memory.

In this study, we investigate the extent that value influences item and context memory (as measured objectively and subjectively) for higher-value relative to lower-value materials. Across two experiments, participants studied words associated with different values at encoding. Values associated with words were presented either before or after presentation of to-be-learned stimuli (i.e., order effects). Given that we aimed to assess subjective context memory for various episodic details using MCQ, stimuli were presented with various visual and auditory attributes. Specifically, words were presented to participants in various font styles, font colors, and were presented auditorily in either a feminine or masculine voice. After encoding, participants' memory for studied materials was assessed using three measures: item memory (word recognition), an objective measure of context memory (source memory for words spoken in either a feminine or masculine voice), and subjective measures of context memory ( $M C Q$ for visual and auditory details as well as self-generated thoughts and feelings induced by items). We make four predictions: First, for item memory, we expect to see better memory for words associated with higher relative to lower values, consistent with past work (Castel, 2005; Castel et al., 2002; Castel et al., 2007; Castel et al., 2013; Hargis \& Castel, 2017; Hayes et al., 2013; Middlebrooks et al., 2017; Middlebrooks et al., 2016). Second, for the effect of order (whether value is presented before or after an item is presented), we predict better item memory for higher-value relative to lower-value items regardless of whether value is presented before or after the studied word. Such a finding would be consistent with past work (Hayes et al., 2013), and consistent with the idea that prioritization processes are similar whether value is known immediately before or after an episode. Third, for objective context memory, we see one of two possible outcomes. If studying materials associated with higher-value leads to heightened memory for various episodic details as some work suggests (Cohen et al., 2017, Experiments 3-5; Siegel \& Castel, 2018a, Experiment 1), then better context memory should be evident for highervalue relative to lower-value words. Alternatively, if greater focus on higher-value items comes at a cost to encoding contextual details (item-context trade-offs), it may be that item and objective context memory show different patterns, where item memory improvements are not accompanied by similar improvements in context memory, as other work has shown (Castel et al., 2007, Experiments 1-2; Hennessee et al., 2017; Hennessee et al., 2018, Experiment 3; Hennessee et al., 2019, Experiment 1; Stefanidi et al., 2018 , Experiment 4). Given that past works suggest prioritizing information in memory relies on controlled use of resources (i.e., top-down processes) at encoding (Castel et al., 2009; Castel et al., 2011; Robison \& Unsworth, 2017), it may be that VDR effects are evident for some details such as item memory, but not others, such as objective context. Fourth, for subjective context memory (and similar to objective context memory), we expected one of two results: either that item and subjective context would show similar increases in memory for higher-value relative to lower-value items, or that item memory would improve, whereas subjective context would not. Because our subjective memory measure accounts for a range of details (visual, auditory, thoughts and feelings), results will provide a richer understanding of the influence of value on the contents of episodic memory. 


\section{Experiment 1}

\section{Method}

\section{Participants}

Seventy-five undergraduates $(M=19.70, S D=1.94$, range: 18-35, 37 females) were recruited from the University of Illinois at Chicago to participate in this experiment. Three participants did not complete all experimental procedures, and thus were removed from analyses. We used $\mathrm{G}^{*}$ Power (Faul, Erdfelder, Lang, \& Buchner, 2007) to perform an a priori power analysis to determine the sample size needed to detect a significant effect. Based on a previous report for context memory in a VDR paradigm (Cohen et al., 2017), we determined that 60 participants would yield sufficient power (.90) to detect value effects. We obtained informed consent from all participants in accordance with the Institutional Review Board at the University of Illinois at Chicago. Participants received course credit for participating.

\section{Stimuli}

A total of 144 words (72 positive and 72 negative valence adjectives) from Leshikar et al. (2015) were used as stimuli in this experiment. Words ranged from three to ten characters $(M=7.55$ characters, $S D=1.60)$. All 144 words were recorded in both a feminine and masculine voice. Words were counterbalanced across participants to be spoken in either a feminine or masculine voice, presented in either red or green font color, presented in either Comic Sans or Times New Roman font. Because we included an item recognition test in our procedures, words were also counterbalanced to appear as studied items at encoding or as novel items at test. Across participants, words were randomly assigned different values. Words were presented on a monitor in 24-point font on a black background. Participants were seated two feet away from the monitor and were provided headphones with single-use sanitary ear caps to listen to the spoken stimuli. Headphone volume was adjusted prior to beginning the experiment and maintained for the duration of the experiment.

\section{Procedure}

Participants were tested individually in cubicles in a quiet room. There were two phases of the experiment - an encoding (study) phase and a retrieval (test) phase. Before starting the experiment, participants were given instructions and practice trials for both the encoding and retrieval phases of the experiment to ensure participants understood task instructions.

There were three encoding and retrieval blocks, respectively. Encoding and retrieval blocks were interleaved such that participants completed one encoding block before completing a retrieval block. During encoding, participants studied 96 words ( 32 words per encoding block; equal numbers of positive and negative words per block). Each word was associated with a value ranging from 1 (lowest value) to 8 (highest value). In each encoding block, there were two trials associated with each respective value (e.g., two trials associated with a value of 1 , two trials associated with a value of 2 , etc.). For half of the words, value was presented immediately before the word (value-before trials), and for the other half, value was presented immediately after the word (value-after trials; see Fig. 1). Within an encoding block, participants completed 16 consecutive trials where value either came before or after the presentation of the to-be-learned stimulus. Participants were told that they would earn the value associated with the word if they correctly remembered that word at retrieval, as done before (Hennessee et al., 2017; Hennessee et al., 2018; Schwartz et al., 2020). Further, instructions emphasized that participants should try to remember as many words as possible, but also to prioritize remembering higher-value words. For each encoding trial, participants saw a single word presented on screen for 5,000 ms. Words were presented in either red or green font and in Comic Sans or Times New Roman. While the word appeared on the computer screen, the word was simultaneously presented auditorily in either a feminine or masculine voice. To help ensure participants were attending to each word (especially lower-value words), participants were asked to judge whether the word had more than one syllable by pressing either the "1" (one syllable) or "2" key (more than one syllable). ${ }^{1}$ Each encoding trial was separated by a white central fixation cross presented for $250 \mathrm{~ms}$.

The retrieval phase consisted of 96 old words seen at encoding and 48 novel words presented over three retrieval blocks. In each retrieval block, participants made a series of memory judgments for 48 words (16 value-before words studied at encoding, 16 value-after words studied at encoding, and 16 novel words). For each retrieval trial, a word was shown in white font. Participants were given 7,000 ms to judge whether the word was old, new, or whether they did not know $(1=$ old, 2 $=$ new, 3 = don't know). This judgment served as the item memory response. Participants were instructed that they would receive the amount of points associated with that word (at encoding) for each correctly remembered old item, but that they would be penalized four points if they endorsed a novel word as old. We added this penalty as a means to reduce use of a strategy to respond to all words as "old" to increase their point

\footnotetext{
${ }^{1}$ Because we were interested in examining the effects of value and order (value before, value after) on memory, we saw it as essential to include an orienting task to induce participant attention to all stimuli on a trial-by-trial basis. Without such a task, it is possible that participants could elect to simply ignore lower-value stimuli in the value before condition, as a way to improve memory for higher value items, which would impede our ability to truly measure memory effects in this experiment. Given this possibility, we chose to use an orienting task (syllable task) to make it more likely participants were attending to all trials.
} 


\section{Experiment 1}

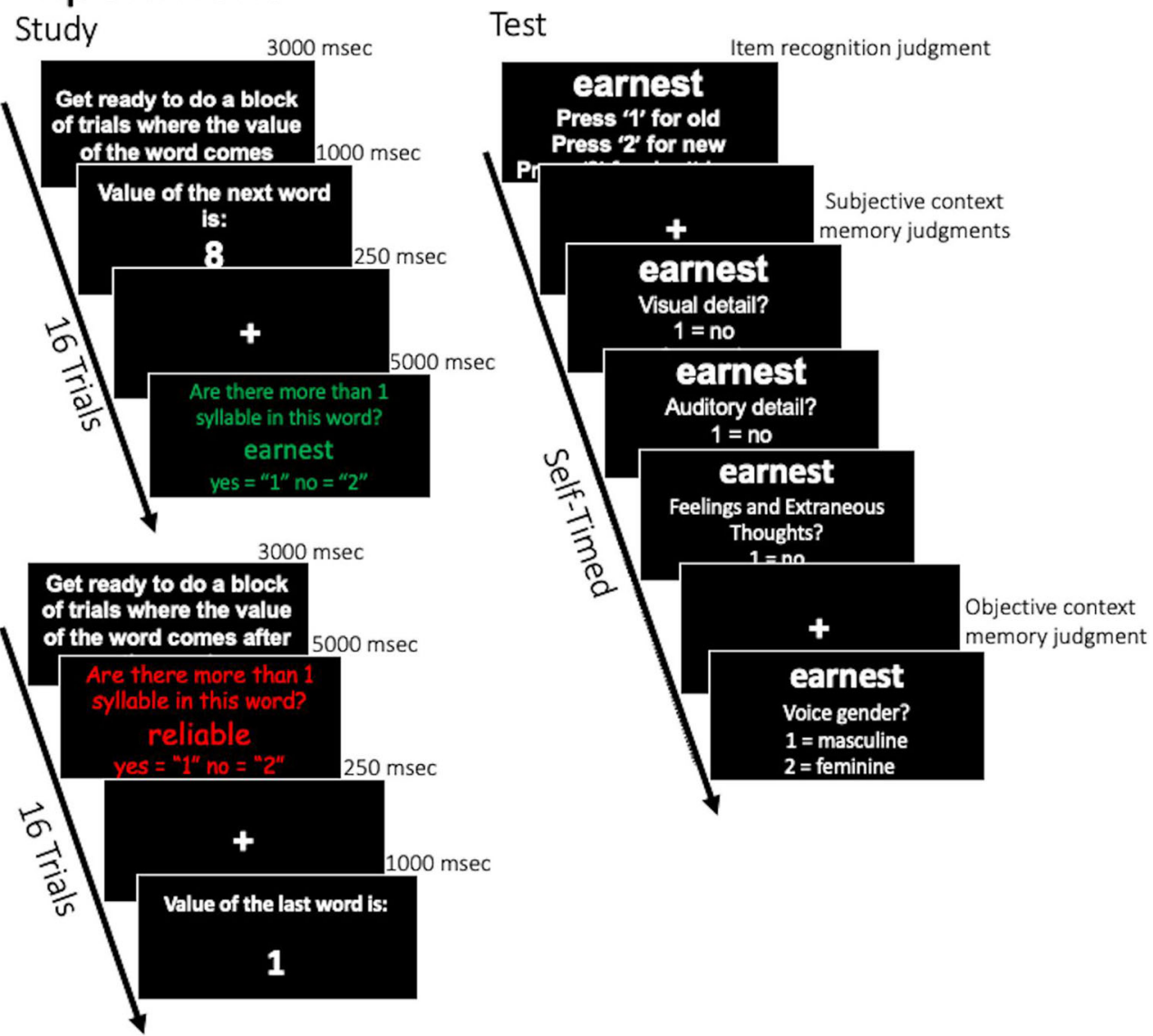

Fig. 1 Trial schematics for the study (encoding) and retrieval (test) phases of Experiment 1

score, as done by others (Cohen et al., 2019; Hennessee et al., 2019; Schwartz et al., 2020). When a word was endorsed as "new," the trial ended and the next retrieval trial began. For words judged as "old," participants were then asked four additional questions for that word that corresponded to subjective and objective context memory. The first three questions were the subjective context memory judgments (i.e., MCQ), where participants were asked to report the amount of details they could retrieve for visual, auditory, and extraneous thoughts and feelings, respectively. For each MCQ question, participants rated the amount of details they could retrieve on a 1-3 scale (1 $=$ no detail remembered, $2=$ few details remembered, $3=$ a lot of details remembered; see Fig. 1). Participants were then asked to make an objective context memory response (source). Participants reported whether the word was presented in a masculine or feminine voice $(1=$ masculine, $2=$ feminine $){ }^{2}$ After

\footnotetext{
${ }^{2}$ In designing this experiment, we considered expanding our objective measures to include other objective contextual details (font type, font color); however, we elected against this procedure because we did not want the retrieval phase to be unduly burdensome to the participants. Given that participants were already making many retrieval judgments for each retrieval trial, we decided to focus on only one objective context measure (source memory).
}

each retrieval block, participants were given feedback on how many points they earned for that block.

\section{Data analysis}

Memory was assessed using three different measures: item, subjective context (MCQs), and objective context memory (source). For all measures (item, subjective context, objective context), memory for studied items was computed as a function of value collapsed into three discrete levels: higher-value items ranged from 7 to 8 points, mediumvalue items ranged from 3 to 6 points, and lower-value items ranged from 1 to 2 points, consistent with past work (Ariel \& Castel, 2014; Hargis \& Castel, 2017; Middlebrooks, McGillivray, Murayama, \& Castel, 2016). For item memory, we computed a corrected measure of item recognition by subtracting the false alarm rates from the hit rates. For subjective context, we analyzed the mean MCQ ratings for visual, auditory, and thoughts and feelings, respectively. Specifically, we calculated the average rating across all participants for each detail type (visual, auditory, thoughts, and feelings), which is analogous to 
our prior work (Leshikar et al., 2015). ${ }^{3}$ For objective context memory, we calculated a corrected measure of source memory (source correct/source correct + source incorrect), as we have done before (Leshikar, Cassidy, \& Gutchess, 2016; Leshikar \& Duarte, 2014; Leshikar, Park, \& Gutchess, 2015; Leshikar \& Gutchess, 2015; McCurdy, Leach, \& Leshikar, 2017). For all our memory measures (item, subjective context, objective context), we computed 3 (value: higher, medium, lower) $\times 2$ (order: value before, value after) analyses of variance (ANOVAs). ${ }^{4}$ For all follow-up comparisons in our ANOVA results, we used Sidak corrections to control for multiple comparisons.

\section{Results}

In this section, we report our memory results (item, subjective context, objective context). Mean responses for studied and novel items are presented in Table 1 as a function of value (higher, medium, lower) and order (value before, value after). Starting first with item memory, results indicated a significant main effect of value, $F(2,142)=6.56, p=.002, \eta_{\mathrm{p}}{ }^{2}=.09$ (see Fig. 2). Follow-up comparisons showed item memory was significantly better for higher-value $(M=0.53, S E=0.02)$ compared with medium-value items $(M=0.47, S E=0.02)$, $p=.001,95 \%$ CI $[.018, .090]$, but higher-value items did not differ from lower-value items $(M=0.50, S E=0.02), p=.26$, $95 \%$ CI $[-0.013, .071]$. Medium-value items did not significantly differ from lower-value items, $p=.14,95 \%$ CI $[-0.055$, $.005]$. The main effect of order and the Value $\times$ Order interaction were not significant, order effect, $F(1,71)=0.06, p=$ $.82, \eta_{\mathrm{p}}^{2}=.00 ;$ Value $\times$ Order interaction, $F(2,142)=0.10, p=$ $.90, \eta_{\mathrm{p}}^{2}=.00 .^{5}$

Because we had a continuous range of values (1-8) in our stimuli, we ran an additional analysis to assess whether item

\footnotetext{
${ }^{3}$ Since participants were using a range of 1 (no details) to 3 (a lot of details) for MCQ ratings, the mean scores for each subjective contextual detail (visual, auditory, thoughts, and feelings) should be interpreted with this range in mind. For instance, if the mean rating for visual details is around 2, this translates into participants subjectively reporting they can retrieve a few visual details for a given trial type. In this example, this could translate to participants remembering a few visual details (like font color), but not other visual details.

${ }^{4}$ Although our stimuli had a valence component (positive, negative adjectives), we did not design this experiment to look at the factor of valence in our primary ANOVA analyses, and thus Experiment 1 was not powered to run analyses including valence. However, to evaluate whether valence affected memory data, we performed a paired-samples $t$ test comparing item memory for valence. Results showed no significance difference for positive words $(M=$ $0.45, S D=0.11)$ relative to negative words $(M=0.45, S D=0.11), t(71)=$ $-.33, p<.74, d=-.04$.

${ }^{5}$ We also calculated item memory using the signal detection measure $d$, which takes into account both item hits as well as item false alarms (Snodgrass \& Corwin, 1988). We entered $d^{\prime}$ scores into a 3 (value: higher, medium, lower) $\times 2$ (order: value before, value after) ANOVA, as in our primary analysis. Results of this analysis fully replicated our primary analysis (based on hits - false alarms): we observed a value effect, $F(2,142)=6.37, p=$ $.005, \eta_{\mathrm{p}}^{2}=.11$, but no effect of order, $F(1,71)=0.12, p=.78, \eta_{\mathrm{p}}{ }^{2}=.00$, or interaction, $F(2,142)=0.45, p=.64, \eta_{\mathrm{p}}^{2}=.01$.
}

memory improved as value increased. To do so, we ran a multilevel mixed-effects logistic regression to analyze the effect of value (1-8) and order (value before, value after) on item memory for the old (studied) items. All mixed-effects models were conducted using jamovi software, specifically the GAMLj module (Gallucci, 2019) for testing mixedeffects generalized linear models. ${ }^{6}$ In this analysis, item accuracy was coded as 0 for incorrect and 1 for correct trials. Value and order were included as fixed effects, whereas subjects were included as random effects (random intercept per subject and random slope per subject for value). Controlling for these random effects of subjects allows us to be confident that any significant predictors are not due to individual differences in overall memory performance and individual differences in the effect of word value across participants (i.e., participants using different strategies as a function of value). Results showed a significant effect of value on item memory, $\chi^{2}(1)=5.00, p=$ .025 , which is consistent with our ANOVA results. Specifically, the higher the word value, the more likely participants were to make accurate recognition judgments, $b=$ .03 . Order did not significantly affect item accuracy judgments, $\chi^{2}(1)<.001, p=.98$. In a subsequent model, we included an interaction term to assess whether word value and condition jointly affected item memory accuracy. The interaction term was not significant, $\chi^{2}(1)=7.85, p=.35$, but the pattern of significance for value remained, value effect, $\chi^{2}(1)$ $=32.24, p<.001$; order effect, $\chi^{2}(1)=.001, p=.97$.

For subjective context memory, mean responses for MCQs are presented in Table 1 as a function of value (higher, medium, lower) and order (value before, value after). We ran separate ANOVAs on each respective MCQ measure (visual, auditory, thoughts and feelings), as done before (Leshikar et al., 2015). Our subjective memory findings are presented in Fig. 3. For visual details, there were no significant effects or interaction, value effect, $F(2,142)=0.73, p=.49, \eta_{\mathrm{p}}{ }^{2}=.01$; order effect, $F(1,71)=3.72, p=.06, \eta_{\mathrm{p}}{ }^{2}=.05$; Value $\times$ Order interaction, $F(2,142)=1.49, p=.23, \eta_{\mathrm{p}}{ }^{2}=.02$.

For auditory details, there was a significant main effect of value, $F(2,142)=3.26, p=.04, \eta_{\mathrm{p}}{ }^{2}=.04$; however, follow-up comparisons controlling for multiple comparisons (Sidak) showed that higher-value items $(M=2.38, S E=0.06)$ did not significantly differ from either medium-value, $(M=$ 2.33, $S E=0.06), p=.06,95 \%$ CI $[-0.001, .095]$, or lowervalue items $(M=2.32, S E=0.06), p=.13,95 \%$ CI $[-0.011$, .120]. Further, medium-value items did not differ from lowervalue items, $p=.98,95 \% \mathrm{CI}[-0.047, .061]$. The main effect of order and interaction were not significant, order effect, $F(1$,

\footnotetext{
${ }^{6}$ The model employed an effect coding scheme for order, which allowed us to set the value-before trials as the reference group with which to compare with value-after trials. This effect coding scheme allows for easier interpretation of any interaction effects, compared to dummy/treatment coding (Singmann \& Kellen, 2019).
} 
Table 1 Item recognition and objective context (source memory) responses, as well as mean subjective context (MCQ) ratings are presented as a function of value (higher, medium, lower) and order (value before, value after) for Experiment 1 (memory responses for novel [unstudied] items are also reported)

\begin{tabular}{|c|c|c|c|c|c|c|c|c|c|c|c|c|}
\hline \multirow[t]{2}{*}{ Value range } & \multicolumn{6}{|c|}{ Value before } & \multicolumn{6}{|c|}{ Value after } \\
\hline & \multicolumn{2}{|c|}{ Higher } & \multicolumn{2}{|c|}{ Medium } & \multicolumn{2}{|c|}{ Lower } & \multicolumn{2}{|c|}{ Higher } & \multicolumn{2}{|c|}{ Medium } & \multicolumn{2}{|c|}{ Lower } \\
\hline \multicolumn{13}{|l|}{ Memory for item } \\
\hline Studied items & $M$ & $S D$ & $M$ & $S D$ & $M$ & $S D$ & $M$ & $S D$ & $M$ & $S D$ & $M$ & $S D$ \\
\hline Item hits & 0.73 & $(0.20)$ & 0.66 & $(0.16)$ & 0.67 & $(0.20)$ & 0.71 & $(0.19)$ & 0.66 & $(0.19)$ & 0.69 & $(0.19)$ \\
\hline Item misses & 0.23 & $(0.20)$ & 0.28 & $(0.17)$ & 0.29 & $(0.20)$ & 0.24 & $(0.18)$ & 0.29 & $(0.18)$ & 0.27 & $(0.19)$ \\
\hline Item don't know & 0.04 & $(0.04)$ & 0.06 & $(0.05)$ & 0.04 & $(0.07)$ & 0.05 & $(0.06)$ & 0.05 & $(0.06)$ & 0.04 & $(0.05)$ \\
\hline Unstudied items & $M$ & $S D$ & & & & & & & & & & \\
\hline Correct rejection & 0.76 & $(0.17)$ & & & & & & & & & & \\
\hline False alarm & 0.17 & $(0.16)$ & & & & & & & & & & \\
\hline Don't know & 0.07 & $(0.04)$ & & & & & & & & & & \\
\hline \multicolumn{13}{|c|}{ Memory for objective context (source) } \\
\hline Studied items & $M$ & $S D$ & $M$ & $S D$ & $M$ & $S D$ & $M$ & $S D$ & $M$ & $S D$ & $M$ & $S D$ \\
\hline Source hits & 0.52 & $(0.15)$ & 0.52 & $(0.09)$ & 0.49 & $(0.15)$ & 0.52 & $(0.14)$ & 0.52 & $(0.09)$ & 0.52 & $(0.14)$ \\
\hline Source misses & 0.48 & $(0.15)$ & 0.48 & $(0.09)$ & 0.51 & $(0.15)$ & 0.48 & $(0.14)$ & 0.48 & $(0.09)$ & 0.48 & $(0.14)$ \\
\hline \multicolumn{13}{|c|}{ Memory for subjective context (MCQ) } \\
\hline Studied items & $M$ & $S D$ & $M$ & $S D$ & $M$ & $S D$ & $M$ & $S D$ & $M$ & $S D$ & $M$ & $S D$ \\
\hline Visual details & 2.21 & $(0.59)$ & 2.16 & $(0.55)$ & 2.14 & $(0.55)$ & 2.20 & $(0.55)$ & 2.20 & $(0.54)$ & 2.21 & $(0.57)$ \\
\hline Auditory details & 2.37 & $(0.54)$ & 2.32 & $(0.51)$ & 2.35 & $(0.50)$ & 2.39 & $(0.51)$ & 2.34 & $(0.52)$ & 2.30 & $(0.56)$ \\
\hline Thoughts and feelings & 1.89 & $(0.64)$ & 1.88 & $(0.64)$ & 1.89 & $(0.66)$ & 1.88 & $(0.61)$ & 1.88 & $(0.63)$ & 1.88 & $(0.66)$ \\
\hline
\end{tabular}

$71)=0.08, p=.77, \eta_{\mathrm{p}}{ }^{2}=.00 ;$ Value $\times$ Order interaction, $F(2$, $142)=1.50, p=.23, \eta_{\mathrm{p}}{ }^{2}=.02$.

For thoughts and feelings, results showed no main effects (or interaction) for value or order (value effect: $F(2,142)=$ $0.01, p=.99, \eta_{\mathrm{p}}{ }^{2}=.00$; order effect: $F(1,71)=0.86, p=.36$, $\eta_{\mathrm{p}}{ }^{2}=.01$; Value $\times$ Order interaction: $F(2,142)=0.30, p=.74$, $\eta_{\mathrm{p}}^{2}=.00$.

Turning to objective context memory (source memory), mean responses are presented in Table 1 as a function of value (higher, medium, lower) and order (value before, value after). Results showed no significant main effects or interaction, value effect, $F(2,142)=0.86, p=.43, \eta_{\mathrm{p}}{ }^{2}=.01$; order effect, $F(1$, $71)=0.71, p=.40, \eta_{\mathrm{p}}{ }^{2}=.01$; Value $\times$ Order interaction, $F(2$, $142)=1.15, p=.32, \eta_{\mathrm{p}}^{2}=.02$; see Fig. 4$)^{7,8}$

\footnotetext{
$\overline{7}$ Because we had a continuous range of values (1-8) in our stimuli, we ran an additional analysis to assess whether objective context memory improved as value increased. To do so, we conducted a logistic regression. Value (1-8) and order (value before, value after) were included as predictors. Results showed that memory did not differ as a function of value, $\chi^{2}(1)=.37, b=.008, p=.54$, or order, $\chi^{2}(1)=.09, p=.76$. In a subsequent model, we included an interaction to assess whether value and order jointly affected performance. The interaction term was not significant, $\chi^{2}(7)=3.71, p=.81$, and the main effects remained nonsignificant.

${ }^{8}$ Because performance on our objective context measure was close to chance, we performed an analysis comparing objective context memory with chancelevel performance. Results showed that only trials associated with mediumvalue items were significantly above chance, $t \mathrm{~s}>1.75, p \mathrm{~s}<.05$.
}

\section{Experiment 1 discussion}

We investigated the extent that value influenced item memory as well as both subjective and objective context memory depending on whether value was presented before or after an item was studied. Results indicated item memory value effects, but we did not find evidence that value influenced context memory (either subjective or objective). Finding item memory value effects is consistent with many previous investigations (Castel et al., 2013; Elliott et al., 2020; Hennessee et al., 2019; Middlebrooks et al., 2017) and highlights the notion that participants are readily able to prioritize learning valuable materials. Interestingly, although we did not find that item memory was better for higher-value relative to lowervalue items, results of the logistic regression showed clear evidence of item memory value effects, where item memory improved with each increase in value. Further, finding that item memory was lowest for medium-value items (e.g., memory for medium-value items was numerically reduced relative to lower-value items) was not expected; however, a closer look through existing VDR literature does sometimes show this pattern (where lower-value items show better memory than medium-value items, at least numerically; Castel et al., 2002; Castel et al., 2007; Middlebrooks et al., 2017). Additionally, we did not find evidence for true item-context 


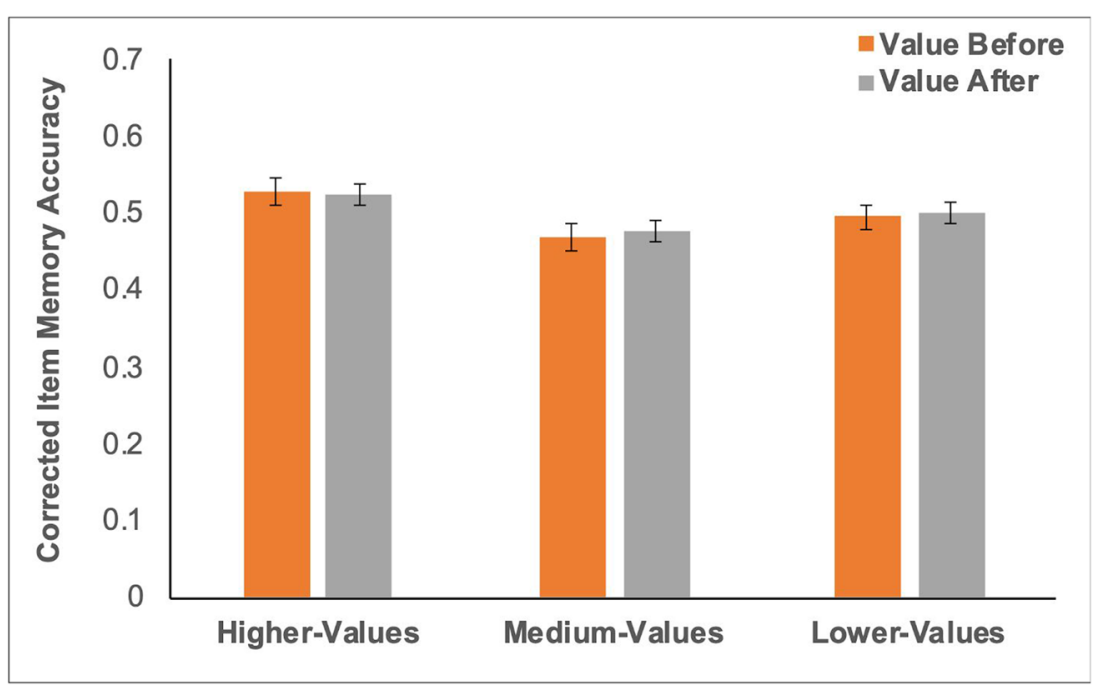

Fig. 2 Mean item memory performance as a function of value and order for Experiment 1. Error bars denote standard error of mean

trade-offs (where memory was enhanced for item, but decreased for context).

Although some past work has shown value effects for both subjective (Cohen et al., 2017; Elliott et al., 2020; Siegel \& Castel, 2018b) as well as objective context memory (Cohen et al., 2017, Experiments 3-5; Siegel \& Castel, 2018a, Experiment 1), we found no such evidence in Experiment 1. The fact that we did not see subjective context memory value effects is particularly surprising, given that past VDR work has consistently shown value effects for subjective context memory as measured by "remember" judgments (Cohen et al., 2017; Elliott \& Brewer, 2019; Hennessee et al., 2017). One possible reason participants did not show value effects for our subjective context memory measures (as measured by MCQ), is that participants may have been challenged to remember the various episodic contextual details associated with each trial (value range of $1-8$, voice source, font color, etc.), which in turn could have made it more difficult to allocate encoding resources (in a top-down manner) to sufficiently remember higher-value versus lower-value materials. Thus, it is possible that with fewer details to encode, participants might be better able to more effectively allocate encoding resources to remember peripheral contextual details associated with valuable materials.

To further investigate value effects on item, subjective, and objective context memory, we conducted an additional experiment. To reduce the context memory burden on participants we reduced the range of valuable items to $1-4$ (instead of 1-8, as in Experiment 1). By reducing the range of values, it is possible that participants are able to more flexibly deploy (top-down) encoding resources since it may be easier to determine what are higher-value versus lower-value items. By reducing the range of value, it may be that participants are better able to use resources to encode richer episodic representations that include contextual details for higher-value relative to lower-value materials.

\section{Experiment 2}

\section{Methods}

\section{Participants}

Seventy-nine undergraduates $(M=19.54, S D=2.70$, range: 17-33, 52 females) were recruited from the University of Illinois at Chicago to participate in this experiment. We obtained informed consent from all participants in accordance with the Institutional Review Board at the University of Illinois at Chicago. Participants received course credit for participating.

\section{Stimuli}

Stimuli were identical to Experiment 1, except that the range of values associated with items was $1-4^{9}$ (instead of $1-8$, as in Experiment 1).

\section{Procedure}

The procedure was identical that of to Experiment 1.

\footnotetext{
${ }^{9}$ In Experiment 2, we chose to associate items with four different values (1-4). Although past VDR studies using few values to associate with items have used values at extreme ends of a scale, such as using only a few higher values (i.e., 7 and 9) and a few lower values (i.e., 1 and 3; Elliott et al., 2020), we elected not to use values at the extreme ends of a value range, because we wanted to replicate our use a continuous range of values as we did in Experiment 1. Further, because some of our ancillary analyses treat value as a continuous variable (logistic regression), using a continuous range of values allows us to perform analogous analyses in Experiment 2, which allows for more direct comparison to the effects identified in Experiment 1.
} 


\section{Memory Characteristics Questionnaire (MCQ)}

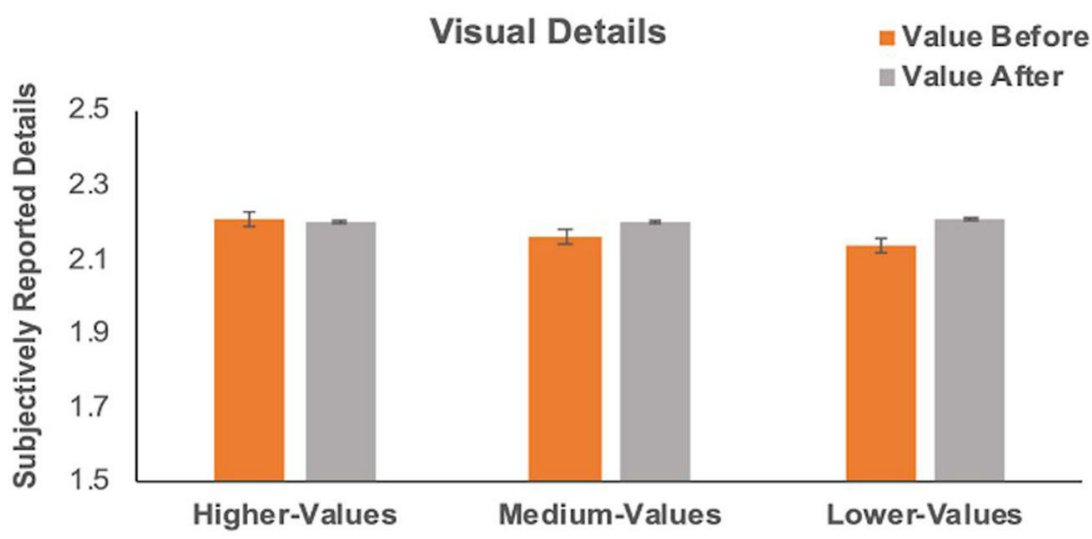

Auditory Details

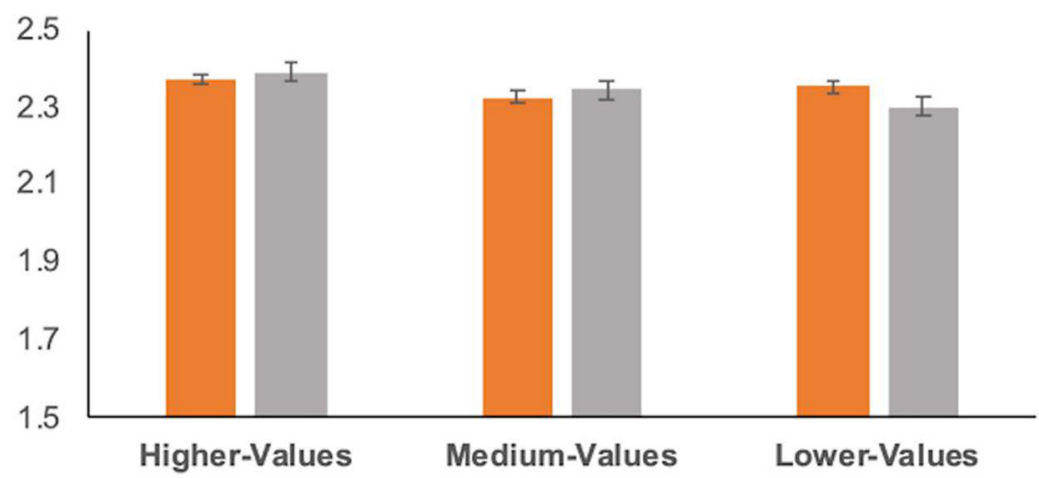

Extraneous Thoughts \& Feelings Details

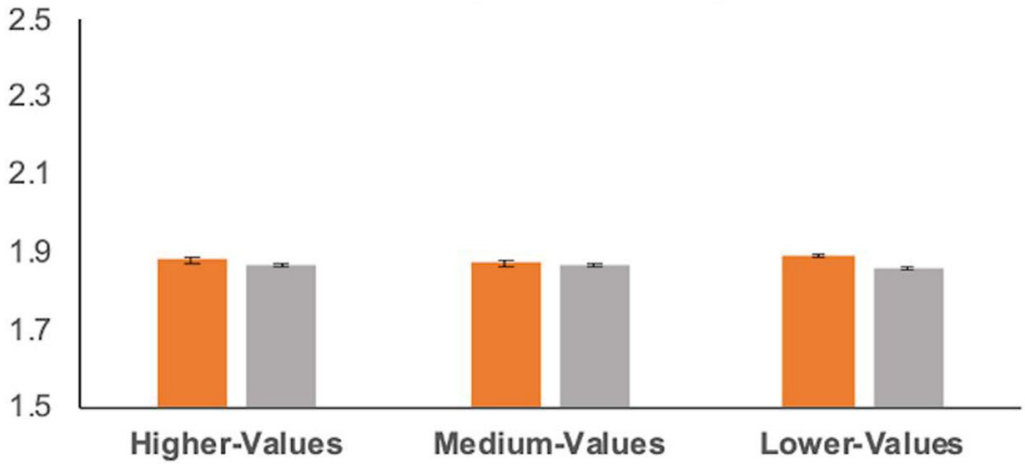

Fig. 3 Mean subjective context memory (MCQs) performance as a function of value and order for Experiment 1. Participants were asked to subjectively rate the "amount" of details $(1=$ none, $2=f e w, 3=a$ lot $)$

\section{Data analysis}

As in Experiment 1, we computed three memory measures: item, subjective context (MCQs), and objective context (source). Trials associated with values of 3-4 were binned as higher-value items, whereas trials associated with 1-2 were binned as lower-value items. For all our memory measures (item, subjective context, they could retrieve for each respective detail type. Error bars denote standard error of mean

objective context), we computed 2 (value: higher, lower) $\times 2$ (order: value before, value after) ANOVAs.

\section{Results}

Mean responses for studied items are presented in Table 2 as a function of value (higher, lower) and order (value before, value 


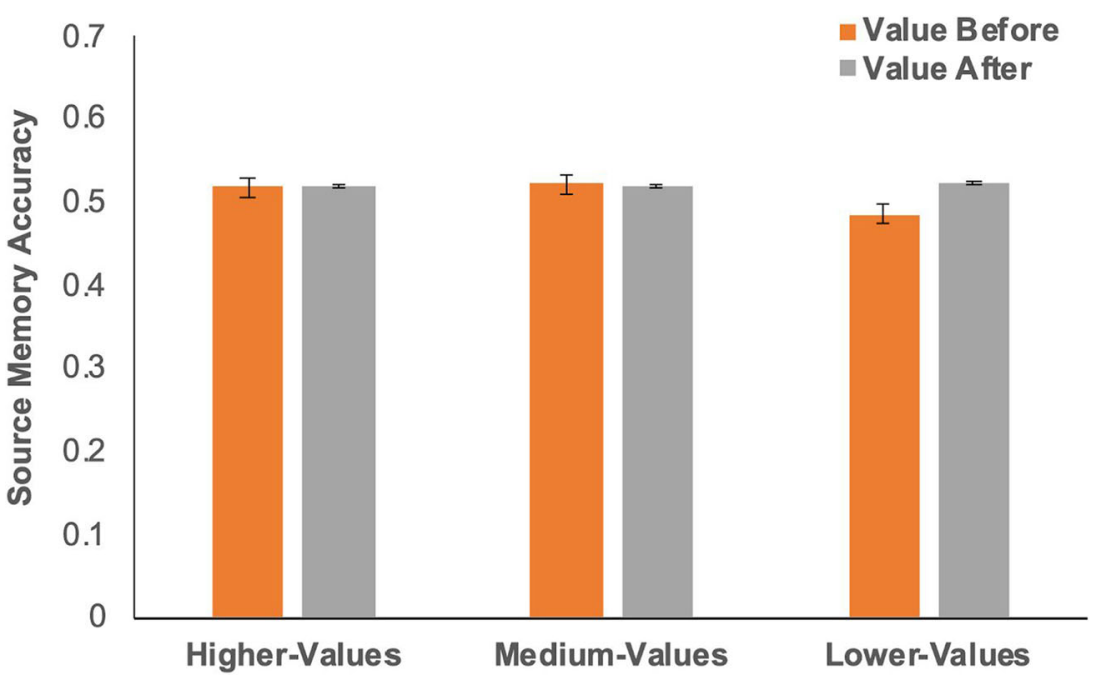

Fig. 4 Mean objective context memory (source) performance as a function of value and order for Experiment 1. Error bars denote standard error of mean

after) as well as the responses to novel items. Starting first with item memory, results indicated a significant Value $\times$ Order interaction, $F(1,78)=5.43, p=.02, \eta_{\mathrm{p}}^{2}=.07$ (see Fig. 5). This interaction was driven by significantly better memory for higher-value $(M=0.50, S E=0.03)$ compared with lower-value words $(M=0.46, S E=0.02)$ when the value was presented before, $t(78)=2.89, p=.005$; however, there was no difference between higher-value $(M=0.47, S E=0.03)$ and lower-value words $(M=0.47, S E=0.03)$ when value was presented after, $t(78)=0.28, p=.78$. The main effects of order and value were not significant, value effect, $F(1,78)=2.11, p=.15, \eta_{\mathrm{p}}{ }^{2}=.03$; order effect, $F(1,78)=1.20, p=.28, \eta_{\mathrm{p}}{ }^{2}=.02$.

As in Experiment 1, we also performed a multilevel mixedeffects logistic regression on item memory, with value (1-4) and order (value before, value after) as fixed effects, and subjects as random effects (random intercept per subject and random slope per subject for value). Item memory did not differ as a function of value, $\chi^{2}(1)=1.53, b=.03, p=.21$, or order, $\chi^{2}(1)=.55, p=.45$. In a subsequent model, we included an interaction to assess whether word value and condition jointly affected item memory accuracy. The interaction term was not significant, $\chi^{2}(1)=5.66$, $p=.13$, and the main effects remained nonsignificant, value, $\chi^{2}(1)=2.44, p=.49$; order, $\chi^{2}(1)=.57, p=.45$.

Our subjective context memory (MCQ) findings are presented in Fig. 6 as a function of value and order. For visual details, there was a significant main effect of value, $F(1,78)=12.56, p=$ $.001, \eta_{\mathrm{p}}{ }^{2}=.14$, which was driven by better memory for highervalue $(M=2.29, S E=0.06)$ compared with lower-value words $(M=2.22, S E=0.06)$. The main effect of order and the Value $\times$ Order interaction were not significant, order effect, $F(1,78)=$ $0.02, p=.90, \eta_{\mathrm{p}}{ }^{2}=.00 ;$ Value $\times$ Order interaction, $F(1,78)=$ $2.55, p=.11, \eta_{\mathrm{p}}^{2}=.03$.

For auditory details, there was a significant main effect of value, $F(1,78)=13.96, p<.001, \eta_{\mathrm{p}}{ }^{2}=.15$, stemming from better memory for higher-value $(M=2.45, S E=0.05)$ compared with lower-value words $(M=2.39, S E=0.05)$. The main effect of order and interaction were not significant, order effect, $F(1,78)=1.89, p=.17, \eta_{\mathrm{p}}{ }^{2}=.02$; Value $\times$ Order interaction, $F(1,78)=1.07, p=.30, \eta_{\mathrm{p}}{ }^{2}=.01$.

For thoughts and feelings, the main effect of value was significant, $F(1,78)=5.72, p=.02, \eta_{\mathrm{p}}{ }^{2}=.07$, driven by better memory for higher-value $(M=2.00, S E=0.07)$ compared with lower-value words $(M=1.94, S E=0.07)$. The main effect of order and Value $\times$ Order interaction were not significant, order effect, $F(1,78)=0.93, p=.34, \eta_{\mathrm{p}}{ }^{2}=.01$; Value $\times$ Order interaction, $F(1,78)=1.55, p=.22, \eta_{\mathrm{p}}{ }^{2}=.02$.

Turning to objective context memory (source memory), mean source memory responses are presented in Table 2 as a function of value (higher, lower) and order (value before, value after). Results showed a significant main effect of order, $F(1,78)=4.60, p=.04, \eta_{\mathrm{p}}{ }^{2}=.06$ (see Fig. 7), denoted by better memory for trials in the value before $(M=0.53, S E=$ $0.01)$ versus value-after condition $(M=0.51, S E=0.01)$. The main effects of value and interaction were not significant, value effect, $F(1,78)=0.95, p=.33, \eta_{\mathrm{p}}{ }^{2}=.01$; Value $\times$ Order interaction, $F(1,78)=0.03, p=.86, \eta_{\mathrm{p}}{ }^{2}=.00 .^{10,11,12}$

\footnotetext{
${ }^{10}$ We also performed a logistic regression on the objective context memory measure, as in Experiment 1. Results showed that memory did not differ as a function of value, $\chi^{2}(1)=.04, b=-.005, p=.84$, or order, $\chi^{2}(1)=1.86, p=$ .17. In a subsequent model, we included an interaction to assess whether value and order affected objective context memory. The interaction term was not significant, $\chi^{2}(3)=.33, p=.95$, and the main effects remained nonsignificant. ${ }^{11}$ We performed an analysis comparing objective context memory with chance level performance. Results showed that only value before trials (higher, lower) were above chance, $t \mathrm{~s}>1.93, p \mathrm{~s}<.05$.

${ }^{12}$ Because we had both positive and negative stimuli in this data, we ran additional 2 (value: higher, lower) $\times 2$ (order: value before, value after) $\times 2$ (valence: positive, negative) ANOVAs for item, objective context, and subjective context memory (visual, auditory, thoughts and feelings). Results showed no significant effects of valence or valence interactions for item, objective context, and two of the subjective context details (visual, auditory), $F \mathrm{~s}$ $<2.60, p \mathrm{~s}>.11$. There was a main effect of valence for thoughts and feelings, $F(1,76)=5.66, p=.02, \eta_{\mathrm{p}}{ }^{2}=.09$, however, which was driven by more subjective reported details for negative $(M=1.99, S E=.07)$ than positive items $(M=1.94, S E=.07)$.
} 
Table 2 Item recognition and objective context (source memory) responses, as well as mean subjective context (MCQ) ratings are presented as a function of value (higher, medium, lower) and order (value before, value after) for Experiment 2 (memory responses for novel [unstudied] items are also reported)

\begin{tabular}{|c|c|c|c|c|c|c|c|c|}
\hline \multirow[t]{2}{*}{ Value Range } & \multicolumn{4}{|c|}{ Value before } & \multicolumn{4}{|c|}{ Value after } \\
\hline & Higher & & Lower & & Higher & & Lower & \\
\hline \multicolumn{9}{|l|}{ Memory for item } \\
\hline Studied items & $M$ & $S D$ & $M$ & $S D$ & $M$ & $S D$ & $M$ & $S D$ \\
\hline Item hits & 0.69 & $(0.20)$ & 0.66 & $(0.18)$ & 0.65 & $(0.21)$ & 0.67 & $(0.21)$ \\
\hline Item misses & 0.26 & $(0.19)$ & 0.27 & $(0.17)$ & 0.29 & $(0.20)$ & 0.28 & $(0.21)$ \\
\hline Item don't know & 0.04 & $(0.07)$ & 0.04 & $(0.06)$ & 0.03 & $(0.06)$ & 0.03 & $(0.06)$ \\
\hline Unstudied items & $M$ & $S D$ & & & & & & \\
\hline Correct rejection & 0.70 & $(0.18)$ & & & & & & \\
\hline False alarm & 0.21 & $(0.16)$ & & & & & & \\
\hline Don’t know & 0.06 & $(0.08)$ & & & & & & \\
\hline \multicolumn{9}{|c|}{ Memory for objective context (source) } \\
\hline Studied items & $M$ & $S D$ & $M$ & $S D$ & $M$ & $S D$ & $M$ & $S D$ \\
\hline Source hits & 0.52 & $(0.11)$ & 0.55 & $(0.11)$ & 0.51 & $(0.13)$ & 0.52 & $(0.09)$ \\
\hline Source misses & 0.48 & $(0.11)$ & 0.45 & $(0.11)$ & 0.49 & $(0.13)$ & 0.48 & $(0.09)$ \\
\hline \multicolumn{9}{|c|}{ Memory for subjective context (MCQ) } \\
\hline Studied items & $M$ & $S D$ & $M$ & $S D$ & $M$ & $S D$ & $M$ & $S D$ \\
\hline Visual details & 2.30 & $(0.50)$ & 2.21 & $(0.54)$ & 2.28 & $(0.51)$ & 2.24 & $(0.56)$ \\
\hline Auditory details & 2.48 & $(0.48)$ & 2.40 & $(0.50)$ & 2.43 & $(0.50)$ & 2.39 & $(0.50)$ \\
\hline Thoughts and feelings & 2.00 & $(0.65)$ & 1.96 & $(0.66)$ & 2.00 & $(0.62)$ & 1.92 & $(0.66)$ \\
\hline
\end{tabular}

\section{Experiment 2 discussion}

In Experiment 2, we reduced the value range associated with items (1-4) to further investigate the effects of value on item, objective context, and subjective context memory. As in Experiment 1, results showed that value had an influence on item memory. Specifically, we found a Value $\times$ Order interaction where memory was better for higher-value compared with lower-value items when value was known before, but not when value was known after. Additionally, we found no evidence that value affected objective context memory consistent with the findings of Experiment 1, and in line with previous work that has failed to find objective context memory value effects (Castel et al., 2007, Experiments 1-2; Hennessee et al., 2017; Hennessee et al., 2018, Experiment 3; Hennessee et al., 2019, Experiment 1; Stefanidi et al., 2018, Experiment 4).

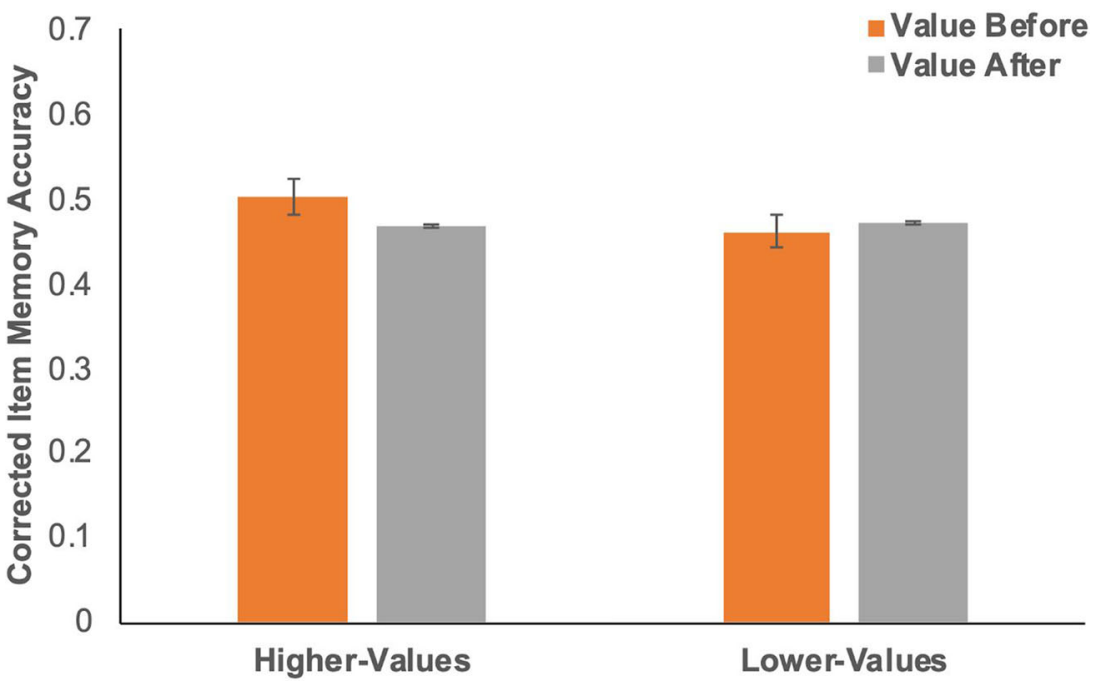

Fig. 5 Mean item memory performance as a function of value and order for Experiment 2. Error bars denote standard error of mean 


\section{Memory Characteristics Questionnaire (MCQ)}
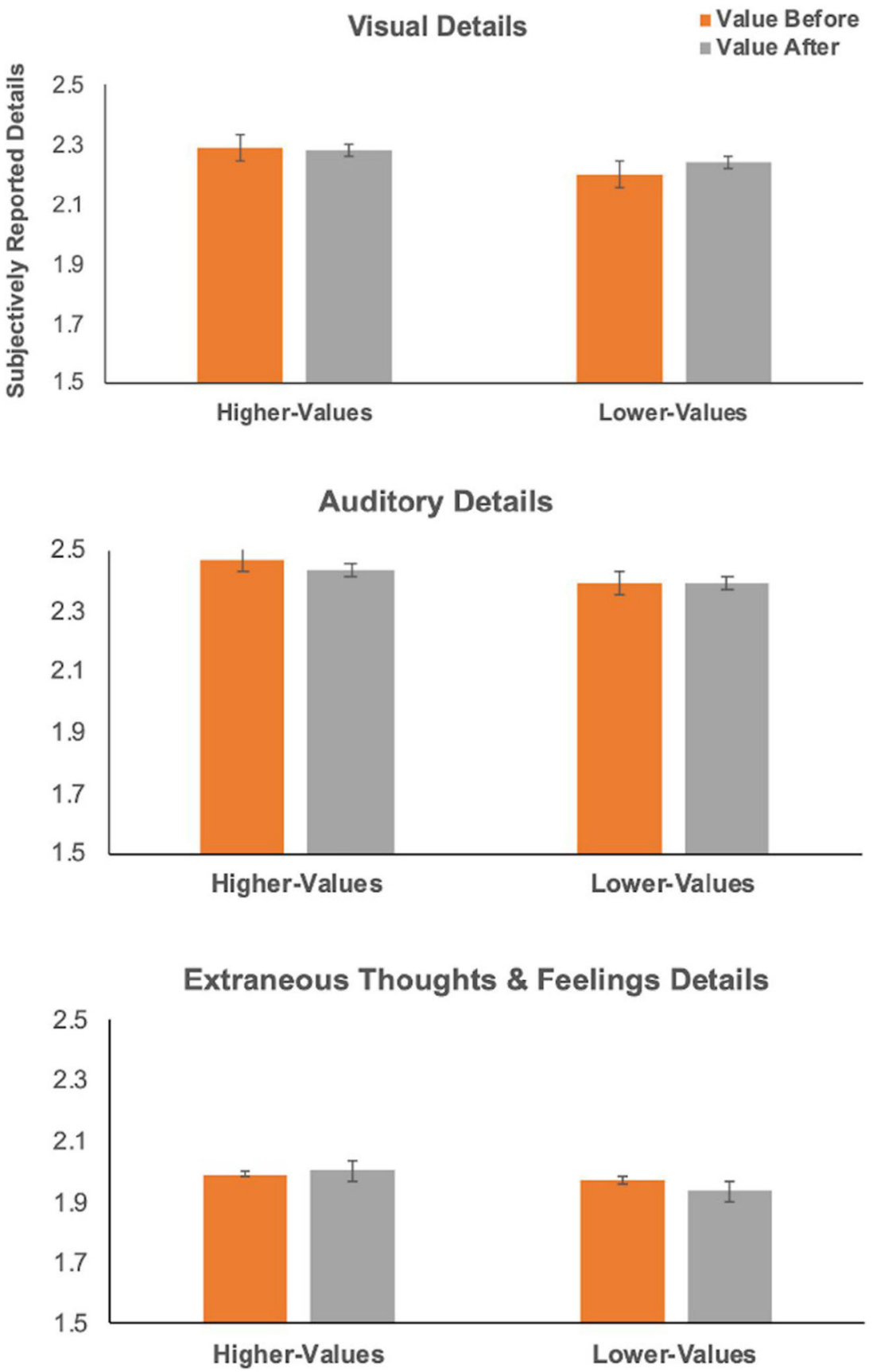

Fig. 6 Mean subjective context memory (MCQs) performance as a function of value and order for Experiment 2. Participants were asked to they could retrieve for each respective detail type. Error bars denote standard error of mean

For subjective context, we found evidence of value effects on subjective memory for visual, auditory, and thoughts and feelings as measured by MCQ. We found these subjective context memory effects under conditions where we reduced the range of values associated with items (1-4) which reduced the context memory burden on participants, and may have enabled easier allocation of encoding resources (in a topdown manner) to remember contextual details associated with valuable items. This evidence suggests that participants are indeed able to show better memory for higher-value items for a variety of episodic details, but only under certain conditions, such as those in Experiment 2. 


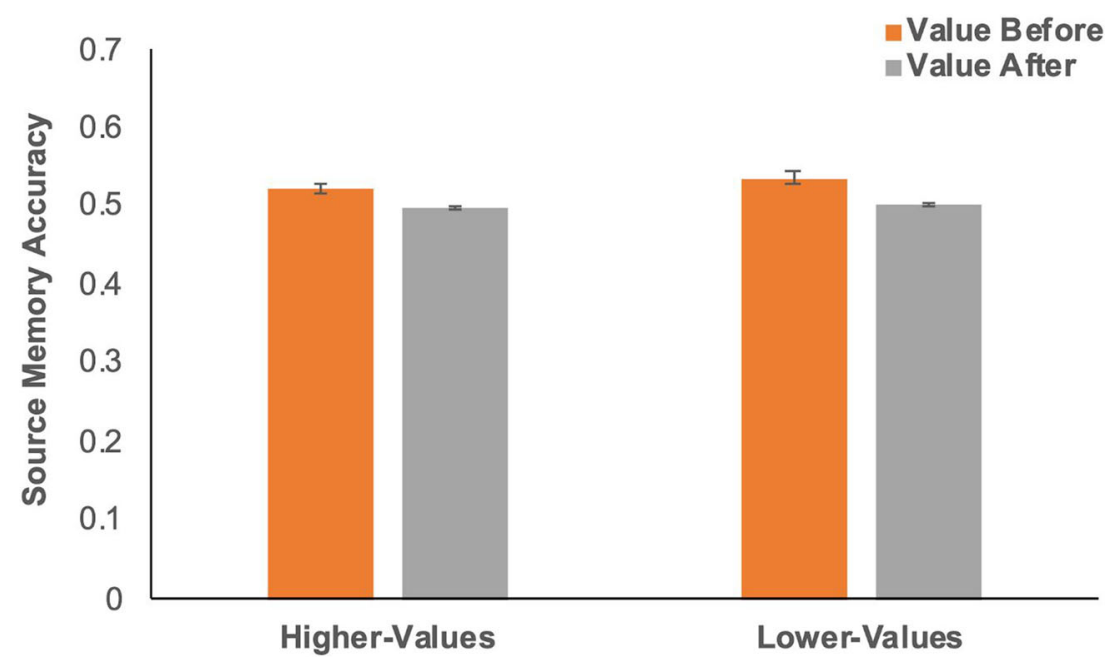

Fig. 7 Mean objective context memory (source) performance as a function of value and order for Experiment 2. Error bars denote standard error of mean

\section{General discussion}

In these experiments, we investigated the extent that value influences item and context memory (as measured subjectively and objectively), and whether the ability to prioritize learning valuable information might differ depending on whether value is known before or after studying an item. There were four main findings in this experiment. First, we found evidence that value influenced item memory in both Experiments 1 and 2. This is consistent with past VDR work demonstrating that people better remember higher-value versus lower-value materials (Castel et al., 2002; Castel et al., 2013). Second, we found relatively little evidence that order (value before versus value after) influenced memory (except for item memory in Experiment 2), which suggest that processes associated with remembering valuable information might be similar whether value is known immediately before or after studying an item. Third, we found no evidence of value effects on objective context memory (source memory) in either experiment. Fourth, we observed some evidence of subjective context memory value effects, but only in Experiment 2, when we reduced the contextual memory burden on participants. Subjective context memory value effects are consistent with past work showing that participants subjectively report remembering more contextual details for higher-value relative to lower-value materials (Cohen et al., 2017; Elliott \& Brewer, 2019; Elliott et al., 2020; Hennessee et al., 2017). Further, we did not observe true item-context trade-offs (where memory was enhanced for item, and decreased for context) in either Experiment 1 or 2, suggesting that participants' ability to encode higher-value items does not come at a cost to encoding of peripheral episodic details. Overall, these data suggest that people can prioritize remembering higher-value content (i.e., item memory), but that this memory benefit extends to contextual details only under some conditions.
Past work has shown that people can prioritize learning valuable or important information relative to information that is less valuable as measured by item memory (Castel, 2005; Castel et al., 2002; Castel et al., 2007; Castel et al., 2013; Hargis \& Castel, 2017; Hayes et al., 2013; Middlebrooks et al., 2017; Middlebrooks et al., 2016). In Experiments 1 and 2 , we examined whether item memory might differ whether value is known before or after to-be-learned items are presented. Results indicated value affected item memory in both Experiments 1 and 2. Interestingly, in Experiment 2, we found item memory value effects driven by better memory for higher-value relative to lower-value materials, but only when value was presented before. The fact that we did not find value effects in the value-after condition in Experiment 2 is in contrast to past work (Hayes et al., 2013), and the results of Experiment 1. It may be that reducing the range of values associated with items (1-4) allowed participants to more readily allocate resources to strategically remember higher-value versus lower-value items, but only when value was known before trials were presented. Further, it is worth noting the logistic regression model did not show a significant Value $\times$ Order interaction, as shown by the ANOVA. One possible reason for this is that we used a corrected measure of item memory (hits - false alarms) in our ANOVA, whereas we only used item memory hits (and misses) in the logistic regression model. Because we applied a correction for false alarms in our ANOVA analysis, it may be that our ANOVA results better reflect participants item memory performance as a function of value. Future work may be necessary to understand how order (value before, value after) affects encoding processes associated with remembering valuable items. Identifying ways to enhance memory is important scientific pursuit (Frankenstein et al., 2021; Giannakopoulos et al., in press; Jennings \& Jacoby, 1993; Leach, McCurdy, Trumbo, Matzen, \& Leshikar, 2018; Leshikar, Duarte, \& Hertzog, 2012; Leshikar et al., 2017; Matzen, Trumbo, Leach, \& 
Leshikar, 2015; Meyers, McCurdy, Leach, Thomas, \& Leshikar, 2020), and the findings of this study add to that endeavor.

Turning to our objective context findings (i.e., source memory), we observed no evidence for memory improvements for higher-value compared with lower-value items in either Experiment 1 or Experiment 2. Although some studies have found a context memory benefit for higher-value compared with lower-value items (Cohen et al., 2019; Stefanidi et al., 2018, Experiment 3), our findings are consistent with other work showing no evidence for context memory advantages (Castel et al., 2007, Experiments 1-2; Hennessee et al., 2017, Experiment 3; Hennessee et al., 2018, Experiment 1; Hennessee et al., 2019, Experiment 1). Past studies have failed to show a context memory value effect for a variety of details, including font color (Hennessee et al., 2017, Experiment 3; Hennessee et al., 2018, Experiment 1; Hennessee et al., 2019, Experiment 1), precise value of word (Castel et al., 2007, Experiment 1-2; Hennessee et al., 2017, Experiment 1; Hennessee et al., 2018, Experiment 1; Hennessee et al., 2019, Experiment 1; Stefanidi et al., 2018, Experiment 4), value range of word (Castel et al., 2007, Experiments 1-2), and grocery prices (Castel, 2005, Experiments 1-2). Overall, the findings of the current study, in conjunction with these past findings, may suggest that people have reduced ability to prioritize learning contextual details, at least those measured objectively (such as voice source). Given the mixed finding of objective context memory in past VDR experiments, it may be that memory for peripheral contextual details can be improved, but these effects are modest and harder to detect relative to item memory. It is worth noting that some investigations that have not observed objective context value effects have used experimental conditions to intentionally deplete encoding resources (Elliott \& Brewer, 2019; Siegel \& Castel, 2018b). For instance, Siegel and Castel (2018b) showed that item location memory (as measured by spatial location error) was not sensitive to value effects under divided attention conditions, and thus our use of the syllable task may have reduced encoding resources available to participants to effectively remember objective contextual details (such as voice source). Another possible reason we did not see value effects for objective context memory may be attributable to the amount of details associated with our stimuli. Specifically, because stimuli were associated with many contextual attributes (a range of values; various visual and auditory details), this may have led to reduced ability to retrieve memory for a specific objective detail (source). Indeed, memory for objective context was near chance, which means that these objective context memory measures should be interpreted cautiously. Future work might further investigate the extent participants are able to encode objective contextual details in tasks where memory performance is not susceptible to possible floor effects.
In addition to objective context, we also examined subjective context memory as a function of value and order. In Experiment 1, analyses showed no evidence of subjective context memory improvements for trials associated with higher-value compared with lower-value items, which is consistent with our objective context memory observation, and further in line with the idea that participants are less able to prioritize remembering contextual details for valuable materials. Interestingly, however, in Experiment 2 (where we reduced the range of values associated with items to $1-4$ ), we found strong evidence of subjective context memory value effects. Specifically, participants subjectively reported more details for higher-value materials in all our MCQ measures (visual, auditory, thoughts and feelings). Finding that participants report retrieving a variety of contextual details (visual, auditory, thoughts and feelings) is consistent with past VDR work showing enhanced rates of subjective "remember" judgments for higher-value relative to lower-value materials (Cohen et al., 2017; Elliott et al., 2020; Hennessee et al., 2019). It may be that participants in those past studies were retrieving a variety of contextual details (perceptual details; self-generated thoughts and feelings) when making remember judgments for higher-value compared with lower-value materials. Thus, our subjective context memory findings in Experiment 2 offer a plausible account of the sort of details participants were retrieving in past VDR studies which showed increased "remember" judgments for higher-value materials. Interestingly, when we examined the influence of valence on our memory measures in a secondary analysis (in line with past work showing that value interacts with valence; Eich \& Castel, 2016), we found that participants subjectively reported more thoughts and feelings details for negative than positive trials in Experiment 2. There is a rich history showing that valence affects memory (Kensinger, 2009), and some work suggests younger adults show a memory advantage for negative compared with positive stimuli (Kensinger \& Corkin, 2003; Ochsner, 2000; Reed, Chan, \& Mikels, 2014). Given that participants sometimes show such a negativity bias in memory, it may not be surprising that participants subjectively reported retrieving more thoughts and feelings details for the negative compared with positive trials, although we also note that valence effects were not evident in our other memory measures (i.e., item, objective context, etc.)

In this set of experiments, we observed effects of value on item memory, but inconsistent effects for our context memory measures, notably for subjective context memory. One possible reason we saw item memory value effects in both experiments may be attributable to our use of the syllable task. In both Experiment 1 and Experiment 2, participants were asked to count the number of syllables in each word, which may have induced more "item-specific" encoding processing (processing that helps to make items distinctive from one another, yielding enhanced memory for the items themselves), which 
could have led to pronounced item memory improvement for higher-value relative to lower-value items. In the broader memory literature, theoretical and experimental work in socalled processing accounts have shown that different tasks at encoding can induce enhanced item-specific processing (leading to improved memory for items) or enhanced relational processing (leading to improved memory for details associated with an item at encoding, such as the value associated with an item; Einstein \& Hunt, 1980; Hunt, 2006; Hunt \& Einstein, 1981) that can influence successful retrieval. Specifically, this work suggests that some encoding tasks can bias participants toward processing aspects of the items themselves (i.e., enhance item-specific processing), or bias participants toward processing the relationship between the item and other episodic features (i.e., enhanced relational processing). Turning back to the current study, it may be that our use of the syllable task induced enhanced item-specific processing (because participants were attending to features of the item; i.e., counting syllables), but that the syllable task had little effect on relational processing at encoding (or perhaps even reduced relational processing). Indeed, past work suggests that encoding tasks can enhance one type of processing (item-specific) while leaving another type (relationalprocessing) relatively unaffected, which is in line with some of our past work in another memory domain (generation effect; McCurdy, Frankenstein, et al., 2020; McCurdy, Frankenstein, Sklenar, \& Leshikar, in press; McCurdy, Leach, \& Leshikar, 2019; McCurdy, Viechtbauer, et al., 2020). Such an outcome could yield the effect we saw in Experiment 1, where item memory showed value effects, whereas context memory did not. Taken together, it is possible the syllable task influenced item memory without promoting relational processing of contextual details, which could have reduced our ability to see value effects for contextual details in these experiments. The fact that we found subjective context memory value effects in Experiment 2, however, suggests that even if our syllable task led to enhanced item-specific processing, this did not fully disrupt our ability to measure value effects on context memory.

Past work on value effects on memory have tried to understand how top-down (e.g., controlled use of resources) versus bottom-up processes (e.g., such as involvement of brain regions associated with processing stimuli that are inherently valuable or rewarding) influence the ability to remember valuable materials. In the current study, participants were given an orienting task at encoding (syllable task), which means participants had less reign to adopt strategies to remember highervalue versus lower-value materials. Given this, it is possible the results of our study may reflect a closer measure of bottom-up processes that influence remembering valuable information than past work that has allowed top-down influences (strategy use) to vary freely across participants. Past neuroimaging work (Adcock, Thangavel, Whitfield-Gabrieli,
Knutson, \& Gabrieli, 2006; Wittmann et al., 2005) has shown that bottom-up processing of rewarding stimuli is partially driven by reward systems of the brain (mesolimbic; dopaminergic systems), and thus, it may that the results of our experiments reflect the contributions of these bottom-up processes while offering more control over variation in top-down strategy differences across participants. Additionally, the fact that participants had reduced ability to freely adopt encoding strategies (because of the syllable task) has implications for possible boundary conditions of value effects on memory. Specifically, it may be that when participants have reduced ability to freely select encoding strategies in VDR tasks, this leads to a reduced ability to prioritize learning objective contextual details (at least voice source, as measured in these experiments). Future work is necessary to pursue this possible boundary condition.

It is worth noting that there is another purpose the syllable task served in these experiments: In the aging and memory literature (Dunlosky \& Hertzog, 1998; Hertzog, McGuire, \& Lineweaver, 1998; Leshikar, Gutchess, Hebrank, Sutton, \& Park, 2010; Naveh-Benjamin, Brav, \& Levy, 2007), and more recently in that for younger adults (Kuhns \& Touron, 2019; Leshikar et al., 2012; Mulligan, Rawson, Peterson, \& Wissman, 2018; Schindler, Schindler, \& Reinhard, 2019; Wenzel \& Reinhard, 2019), evidence suggests that there can be important differences in use of encoding strategies across participants. These lines of work suggest that some participants are "better" at using encoding strategies than others. This is important because such differences in strategy use can interfere with measurement of true memory ability. As an experimental solution to this challenge (that differences in strategy can obscure measurement of true memory ability), past work has shown that providing specific strategies at encoding can help reduce the variation in performance due to use of different encoding strategies across participants, which, in turn, allows for a better measure of memory ability. Thus, by providing a specific encoding task (syllable task) in both Experiment 1 and Experiment 2, it may be that we constrained individual differences in strategy implementation allowing for a stable measure of VDR ability across participants.

One important feature of our study was the inclusion of a condition where value was presented after words were shown (i.e., value-after condition). Although we used the syllable task to make it more likely participants were attending to all trials, it is still possible that participants could have tried to actively forget lower-value words so as to not use resources to encode higher-value words (which would be in line with the principles of directed forgetting; Basden, Basden, \& Gargano, 1993; MacLeod, 1998). Although we did not instruct participants to actively forget items, there is work that has investigated the concept of value-directed forgetting, or the ability to forget materials according to value (Friedman \& Castel, 2011; 
Hennessee et al., 2019, Experiment 1). Interestingly, in this value-directed forgetting work participants show poorer memory for lower-value items under directed remembering compared with directed forgetting instructions, which suggests participants can readily adopt a strategy (e.g., exert topdown influences) to avoid learning material. This is relevant because in our study, item recognition rates for the lowervalue items were essentially equivalent regardless of whether value was presented before or after, suggesting that our participants were not likely adopting a strategy to actively forget lower-value words, which gives greater confidence in the generalizability of our findings.

Although we found item memory value effects and some evidence for subjective context memory value effects (in Experiment 2), there are six limitations of this work worth considering. First, in both experiments, participants were trying to remember valuable information as well as count syllables for each item, thus it is possible that our use of the syllable task may have introduced a dual task element at encoding. Such dual task conditions may have depleted encoding resources, making it more challenging for participants to encode episodic contextual details in line with past work (Elliott \& Brewer, 2019; Siegel \& Castel, 2018b). Given that we found subjective context memory value effects in Experiment 2 that also included the syllable task, however, we see this possibility as less likely. It is also worth noting that unlike true dual tasks where the secondary task is fully unrelated to the primary task (such as making judgments to unrelated stimuli; Elliott \& Brewer, 2019; Middlebrooks et al., 2017; Siegel \& Castel, 2018b), the syllable task we used was directly relevant to processing each item. Further, because participants were making the syllable judgment on all trials, any potential dual task demands should be equivalent across all trials, which means that our main findings on the effects of value on memory should still be meaningful. Second, our use of the syllable task may have also reduced participants' ability to engage in deeper or more elaborative encoding in these experiments. Future work might use a different orienting task (such as making a semantic decision about words) to help ensure that participants are attending to all items, without reducing participants' ability to engage in deeper encoding processes. Third, in our task we used adjectives as the to-be-studied words, which is a departure from past VDR studies that often use nouns as stimuli (Castel et al., 2013; Cohen et al., 2017; Elliott et al., 2020). Since adjectives are typically less concrete than nouns, this may have reduced memory performance overall (Paivio, Khan, \& Begg, 2000), given that past work shows that less concrete materials are typically remembered more poorly (Fliessbach, Weis, Klaver, Elger, \& Weber, 2006; Paivio, Walsh, \& Bons, 1994). It is notable that our use of less concrete stimuli may provide an even more "conservative" measure of value effects on memory than past work, precisely because the stimuli we used were potentially less memorable than more concrete stimuli (nouns) used in past VDR work. Thus, the fact that we found value effects for some of our memory measures, suggests that people can prioritize remembering higher-value items even when those items are harder to remember overall. Fourth, we used emotionally valenced materials (positive and negative adjectives), which is a departure from past work that has used neutral stimuli (Castel et al., 2013; Cohen et al., 2017; Elliott et al., 2020). Although valence did not have a strong effect on most of our memory measures (with the exception of the thoughts and feelings MCQ measure in Experiment 2), future work should replicate the current findings using stimuli that are more neutral. Fifth, as we note above, because our objective context measure was generally close to chance performance, this aspect of the data should be interpreted cautiously. Sixth, as part of our encoding instructions, we explicitly told participants to remember higher-value items, which is a slight departure from some VDR studies that have not stated this directly (and instead allowed participants to determine their own strategy on how to process items as a function of value). It is worth noting however, that because we gave such explicit encoding instruction (e.g., try to remember higher-value items), this represents yet another way in which we reduced possible variation in (top-down driven) use of encoding resources across participants.

Past work has shown that people have the ability to remember higher-value relative to lower-value items. We found evidence of item memory value effects, no evidence of objective context memory value effects, and more mixed effects of value on subjective context memory, which suggests limits to prioritization of contextual information in memory. Given that we did not observe strong evidence of order effects (whether value is presented before or after stimuli are encountered), this further suggests that the processes that guide prioritization of details into memory may be similar regardless of whether value is known immediately before or after an item is encountered. Overall, this work extends knowledge about how people prioritize information in memory, which is an important capability that allows individuals to remember information that is potentially valuable or important.

Open practices statement Data from this report are available upon reasonable request.

\section{References}

Adcock, R. A., Thangavel, A., Whitfield-Gabrieli, S., Knutson, B., \& Gabrieli, J. D. (2006). Reward-motivated learning: mesolimbic activation precedes memory formation. Neuron, 50(3), 507-517. https://doi.org/10.1016/j.neuron.2006.03.036

Ariel, R., \& Castel, A. D. (2014). Eyes wide open: Enhanced pupil dilation when selectively studying important information. Experimental 
Brain Research, 232(1), 337-344. https://doi.org/10.1007/s00221013-3744-5

Basden, B. H., Basden, D. R., \& Gargano, G. J. (1993). Directed forgetting in implicit and explicit memory tests: A comparison of methods. Journal of Experimental Psychology: Learning, Memory, and Cognition, 19(3), 603-616. https://doi.org/10.1037/0278-7393.19. 3.603

Castel, A. D. (2005). Memory for grocery prices in younger and older adults: The role of schematic support. Psychology and Aging, 20(4), 718-721. https://doi.org/10.1037/0882-7974.20.4.718

Castel, A. D. (2007). The adaptive and strategic use of memory by older adults: Evaluative processing and value-directed remembering. Psychology of Learning and Motivation, 48, 225-270. https://doi. org/10.1016/S0079-7421(07)48006-9

Castel, A. D., Balota, D. A., \& McCabe, D. P. (2009). Memory efficiency and the strategic control of attention at encoding: Impairments of value-directed remembering in Alzheimer's disease. Neuropsychology, 23(3), 297-306. https://doi.org/10.1037/ a0014888

Castel, A. D., Benjamin, A., Craik, F., \& Watkins, M. (2002). The effects of aging on selectivity and control in short-term recall. Memory \& Cognition, 30(7), 1078-1085. https://doi.org/10.3758/BF03194325

Castel, A. D., Farb, N. A., \& Craik, F. I. (2007). Memory for general and specific value information in younger and older adults: Measuring the limits of strategic control. Memory \& Cognition, 35(4), 689700. https://doi.org/10.3758/bf03193307

Castel, A. D., Humphreys, K. L., Lee, S. S., Galván, A., Balota, D. A., \& McCabe, D. P. (2011). The development of memory efficiency and value-directed remembering across the life span: A cross-sectional study of memory and selectivity. Developmental Psychology, 47(6), 1553-1564. https://doi.org/10.1037/a0025623

Castel, A. D., Lee, S. S., Humphreys, K. L., \& Moore, A. N. (2011). Memory capacity, selective control, and value-directed remembering in children with and without attention-deficit/hyperactivity disorder (ADHD). Neuropsychology, 25(1), 15-24. https://doi.org/10. 1037/a0020298

Castel, A. D., Murayama, K., Friedman, M. C., McGillivray, S., \& Link, I. (2013). Selecting valuable information to remember: Age-related differences and similarities in self-regulated learning. Psychology and Aging, 28(1), 232-242. https://doi.org/10.1037/a0030678

Cohen, M. S., Cheng, L. Y., Paller, K. A., \& Reber, P. J. (2019). Separate memory-enhancing effects of reward and strategic encoding. Journal of Cognitive Neuroscience, 31(11), 1658-1673. https:// doi.org/10.1162/jocn a 01438

Cohen, M. S., Rissman, J., Hovhannisyan, M., Castel, A. D., \& Knowlton, B. J. (2017). Free recall test experience potentiates strategy-driven effects of value on memory. Journal of Experimental Psychology: Learning, Memory, and Cognition, 43(10), 1581-1601. https://doi.org/10.1037/xlm0000395

Cohen, M. S., Rissman, J., Suthana, N. A., Castel, A. D., \& Knowlton, B. J. (2016). Effects of aging on value-directed modulation of semantic network activity during verbal learning. NeuroImage, 125, 10461062. https://doi.org/10.1016/j.neuroimage.2015.07.079

DeLozier, S., \& Rhodes, M. G. (2015). The impact of value-directed remembering on the own-race bias. Acta Psychologica, 154, 6268. https://doi.org/10.1016/j.actpsy.2014.11.009

Dunlosky, J., \& Hertzog, C. (1998). Aging and deficits in associative memory: What is the role of strategy production?. Psychology and Aging, 13(4), 597-607. https://doi.org/10.1037/0882-7974.13.4.597

Eich, T. S., \& Castel, A. D. (2016). The cognitive control of emotional versus value-based information in younger and older adults. Psychology and Aging, 31(5), 503-512. https://doi.org/10.1037/ pag0000106

Einstein, G. O., \& Hunt, R. R. (1980). Levels of processing and organization: Additive effects of individual-item and relational processing.
Journal of Experimental Psychology: Human Learning and Memory, 6(5), 588-598. https://doi.org/10.1037/0278-7393.6.5.588

Elliott, B. L., \& Brewer, G. A. (2019). Divided attention selectively impairs value-directed encoding. Collabra: Psychology, 5(1), Article 4. https://doi.org/10.1525/collabra.156

Elliott, B. L., McClure, S. M., \& Brewer, G. A. (2020). Individual differences in value-directed remembering. Cognition, 201, Article 104275. https://doi.org/10.1016/j.cognition.2020.104275

Faul, F., Erdfelder, E., Lang, A. G., \& Buchner, A. (2007). G*Power 3: A flexible statistical power analysis program for the social, behavioral, and biomedical sciences. Behavior Research Methods, 39, 175-191. https://doi.org/10.3758/BF03193146

Fliessbach, K., Weis, S., Klaver, P., Elger, C. E., \& Weber, B. (2006). The effect of word concreteness on recognition memory. NeuroImage, 32(3), 1413-1421. https://doi.org/10.1016/j. neuroimage.2006.06.007

Frankenstein, A. N., McCurdy, M. P., Sklenar, A. M., Pandya, R., Szpunar, K. K., \& Leshikar, E. D. (2021). Future thinking about social targets: The influence of prediction outcome on memory. Cognition. https://doi.org/10.1016/j.cognition.2020.104390

Friedman, M. C., \& Castel, A. D. (2011). Are we aware of our ability to forget? Metacognitive predictions of directed forgetting. Memory \& Cognition, 39(8), 1448-1456. https://doi.org/10.3758/s13421-0110115-y

Gallucci, M. (2019). GAMLj: General analyses for linear models [jamovi module]. https://gamlj.github.io/

Giannakopoulos, K., McCurdy, M. P., Sklenar, A. M., Frankenstein, A. N., Urban Levy, P., \& Leshikar, E. D. (in press). Using a less constrained practice test enhances the testing effect. American Journal of Psychology

Hargis, M. B., \& Castel, A. D. (2017). Younger and older adults' associative memory for social information: The role of information importance. Psychology and Aging, 32(4), 325-330. https://doi.org/10. 1037/pag0000171

Hayes, M. G., Kelly, A. J., \& Smith, A. D. (2013). Working memory and the strategic control of attention in older and younger adults. The Journals of Gerontology Series B: Psychological Sciences and Social Sciences, 68(2), 176-183. https://doi.org/10.1093/geronb/ gbs057

Hennessee, J. P., Castel, A. D., \& Knowlton, B. J. (2017). Recognizing what matters: Value improves recognition by selectively enhancing recollection. Journal of Memory and Language, 94(6), 195-205. https://doi.org/10.1016/j.jml.2016.12.004

Hennessee, J. P., Knowlton, B. J., \& Castel, A. D. (2018). The effects of value on context-item associative memory in younger and older adults. Psychology and Aging, 33(1), 46-56. https://doi.org/10. 1037/pag0000202

Hennessee, J. P., Patterson, T. K., Castel, A. D., \& Knowlton, B. J. (2019). Forget me not: Encoding processes in value-directed remembering. Journal of Memory and Language, 106(6), 29-39. https://doi.org/10.1016/j.jml.2019.02.001

Hertzog, C., McGuire, C. L., \& Lineweaver, T. T. (1998). Aging, attributions, perceived control, and strategy use in a free recall task. Aging, Neuropsychology, and Cognition, 5(2), 85-106. https://doi. org/10.1076/anec.5.2.85.601

Hunt, R. R. (2006). The concept of distinctiveness in memory research. In R. R. Hunt \& J. B. Worthen (Eds.), Distinctiveness and memory (p. 3-25). Oxford University Press: New York. https://doi.org/10.1093/ acprof:oso/9780195169669.003.0001

Hunt, R. R., \& Einstein, G. O. (1981). Relational and item-specific information in memory. Journal of Verbal Learning and Verbal Behavior, 20(5), 497-514. https://doi.org/10.1016/S0022-5371(81) 90138-9

James, T., Rajah, M. N., \& Duarte, A. (2019). Multielement episodic encoding in young and older adults. Journal of Cognitive 
Neuroscience, 31(6), 837-854. https://doi.org/10.1162/jocn_a_ 01384

Jennings, J. M., \& Jacoby, L. L. (1993). Automatic versus intentional uses of memory: Aging, attention, and control. Psychology and Aging, 8(2), 283-293. https://doi.org/10.1037/0882-7974.8.2.283

Johnson, M. K., Foley, M. A., Suengas, A. G., \& Raye, C. L. (1988). Phenomenal characteristics of memories for perceived and imagined autobiographical events. Journal of Experimental Psychology: General, 117(4), 371-376. https://doi.org/10.1037/0096-3445.117. 4.371

Jurica, P. J., \& Shimamura, A. P. (1999). Monitoring item and source information: Evidence for a negative generation effect in source memory. Memory \& Cognition, 27(4), 648-656. https://doi.org/10. 3758/BF03211558

Kensinger, E. A. (2009). Emotional memory across the adult lifespan. New York, NY: Psychology Press.

Kensinger, E. A., \& Corkin, S. (2003). Memory enhancement for emotional words: Are emotional words more vividly remembered than neutral words? Memory \& Cognition, 31, 1169-1180. https://doi. org/10.3758/BF03195800

Kuhns, J. M., \& Touron, D. R. (2019). Schematic support increases memory strategy use in young and older adults. Psychology and Aging, 35(3), 397-410. https://doi.org/10.1037/pag0000433

Leach, R. C., McCurdy, M. P., Trumbo, M., Matzen, L. E., \& Leshikar, E. D. (2018). Differential age effects of transcranial stimulation on associative memory in younger and older adults. The Journals of Gerontology. Series B: Psychological Sciences and Social Sciences, 74(7), 1163-1173. https://doi.org/10.1093/geronb/gby003

Leshikar, E. D., Cassidy, B. S., \& Gutchess, A. H. (2016). Similarity to the self influences cortical recruitment during impression formation. Cognitive, Affective, \& Behavioral Neuroscience, 16(2), 302-314. https://doi.org/10.3758/s13415-015-0390-3

Leshikar, E. D., \& Duarte, A. (2014). Medial prefrontal cortex supports source memory for self-referenced materials in young and older adults. Cognitive, Affective, \& Behavioral Neuroscience, 14(1), 236-252. https://doi.org/10.3389/fnhum.2013.00537

Leshikar, E. D., Duarte, A., \& Hertzog, C. (2012). Task-selective memory effects for successful implemented encoding strategies. PLoS ONE, 7(5), Article e38160. https://doi.org/10.1371/journal.pone. 0038160

Leshikar, E. D., Dulas, M. R., \& Duarte, A. (2015). Self-referencing enhances recollection in both young and older adults. Aging, Neuropsychology, and Cognition, 22(4), 388-412.

Leshikar, E. D., \& Gutchess, A. H. (2015). Similarity to the self affects impression memory for others. Journal of Applied Research in Memory and Cognition, 4(1), 20-28. https://doi.org/10.1016/j. jarmac.2014.10.002

Leshikar, E. D., Gutchess, A. H., Hebrank, A. C., Sutton, B. P., \& Park, D. C. (2010). The impact of increased relational encoding demands on frontal and hippocampal function in older adults. Cortex, 46(4), 507-521. https://doi.org/10.1016/j.cortex.2009.07.011

Leshikar, E. D., Leach, R. C., McCurdy, M. P., Trumbo, M., Sklenar, A. M., Frankenstein, A. N., \& Matzen, L. E. (2017). Transcranial direct current stimulation of dorsolateral prefrontal cortex improves recall but not recognition memory. Neuropsychologia, 106(11), 390-397. https://doi.org/10.1016/j.neuropsychologia.2017.10.022

Leshikar, E. D., Park, J. M., \& Gutchess, A. H. (2015). Similarity to the self affects impression memory in younger and older adults. The Journal of Gerontology, Series B: Psychological Sciences and Social Sciences, 70(5), 737-742. https://doi.org/10.1093/geronb/ gbt132

MacLeod, C. M. (1998). Directed forgetting. In J. M. Golding \& C. M. MacLeod (Eds.), Intentional forgetting: Interdisciplinary approaches (pp. 1-57). Mahwah, NJ: Erlbaum.

Matzen, L. E., Trumbo, M., Leach, R. C., \& Leshikar, E. D. (2015). Effects of non-invasive brain stimulation on associative memory.
Brain Research, 1624(10), 286-296. https://doi.org/10.1016/j. brainres.2015.07.036

McCurdy, M. P., Frankenstein, A. N, Sklenar, A. M., \& Leshikar, E. D. (2020). Fewer generation constraints increase the generation effect for item and source memory through enhanced relational processing. Memory, 28(5)598-616. https://doi.org/10.1080/09658211.2020. 1749283

McCurdy, M. P., Frankenstein, A. N, Sklenar, A. M., \& Leshikar, E. D. (in press). Examining the relationship between generation constraint and memory. Memory \& Cognition.

McCurdy, M. P., Leach, R., \& Leshikar, E. D. (2019). Fewer constraints influence generation effect for source memory in younger, but not older adults. Open Psychology, 1(1), 168-184. https://doi.org/10. 1515/psych-2018-0012

McCurdy, M. P., Leach, R. C., \& Leshikar, E. D. (2017). The generation effect revisited: Fewer constraints enhances item and context memory. Journal of Memory and Language, 92, 202-216. https://doi. org/10.1016/j.jml.2016.06.007

McCurdy, M. P., Viechtbauer, W., Sklenar, A. M., Frankenstein, A., \& Leshikar, E. D. (2020). Theories of the generation effect and the impact of generation constraint: A meta-analytic review. Psychonomic Bulletin \& Review, 27, 1139-1165. https://doi.org/ 10.3758/s13423-020-01762-3

Meyers, Z. R., McCurdy, M. P., Leach, R. C., Thomas, A. K., \& Leshikar, E. D. (2020). Effects of survival processing on item and context memory: Enhanced memory for survival-relevant details. Frontiers in Psychology, 11, 2244. https://doi.org/10.3389/fpsyg. 2020.02244

Middlebrooks, C. D., Kerr, T., \& Castel, A. D. (2017). Selectively distracted: Divided attention and memory for important information. Psychological Science, 28(8), 1103-1115. https://doi.org/10.1177/ 0956797617702502

Middlebrooks, C. D., McGillivray, S., Murayama, K., \& Castel, A. D. (2016). Memory for allergies and health foods: How younger and older adults strategically remember critical health information. The Journals of Gerontology Series B: Psychological Sciences and Social Sciences, 71(3), 389-399. https://doi.org/10.1093/geronb/ gbv032

Middlebrooks, C. D., Murayama, K., \& Castel, A. D. (2016). The value in rushing: Memory and selectivity when short on time. Acta Psychologica, 170, 1-9. https://doi.org/10.1016/j.actpsy.2016.06. 001

Mulligan, N. W., Rawson, K. A., Peterson, D. J., \& Wissman, K. T. (2018). The replicability of the negative testing effect: Differences across participant populations. Journal of Experimental Psychology: Learning, Memory, and Cognition, 44(5), 752-763. https://doi.org/ 10.1037/xlm0000490

Naveh-Benjamin, M., Brav, T. K., \& Levy, O. (2007). The associative memory deficit of older adults: The role of strategy utilization. Psychology and Aging, 22(1), 202-208. https://doi.org/10.1037/ 0882-7974.22.1.202

Naveh-Benjamin, M., \& Craik, F. I. (1995). Memory for context and its use in item memory: Comparisons of younger and older persons. Psychology and Aging, 10(2), 284-293. https://doi.org/10.1037/ 0882-7974.10.2.284

Ochsner, K. N. (2000). Are affective events richly recollected or simply familiar? The experience and process of recognizing feelings past. Journal of Experimental Psychology: General, 129, 242-261. https://doi.org/10.1037/0096-3445.129.2.242

Paivio, A., Khan, M., \& Begg, I. (2000). Concreteness and relational effects on recall of adjective-noun pairs. Canadian Journal of Experimental Psychology/Revue canadienne de psychologie expérimentale, 54(3), 149-160. https://doi.org/10.1037/h0087337

Paivio, A., Walsh, M., \& Bons, T. (1994). Concreteness effects on memory: When and why? Journal of Experimental Psychology: 
Learning, Memory, and Cognition, 20(5), 1196-1204. https://oi. org/10.1037/0278-7393.20.5.1196

Reed, A. E., Chan, L., \& Mikels, J. A. (2014). Meta-analysis of the agerelated positivity effect: Age differences in preferences for positive over negative information. Psychology and Aging, 29(1), 1-15. https://doi.org/10.1037/a0035194

Robison, M. K., \& Unsworth, N. (2017). Working memory capacity, strategic allocation of study time, and value-directed remembering. Journal of Memory and Language, 93, 231-244. https://doi.org/10. 1016/j.jml.2016.10.007

Rugg, M. D., Vilberg, K. L., Mattson, J. T., Sarah, S. Y., Johnson, J. D., \& Suzuki, M. (2012). Item memory, context memory and the hippocampus: fMRI evidence. Neuropsychologia, 50(13), 3070-3079. https://doi.org/10.1016/j.neuropsychologia.2012.06.004

Schindler, J., Schindler, S., \& Reinhard, M. A. (2019). Effectiveness of self-generation during learning is dependent on individual differences in need for cognition. Frontline Learning Research, 7(2), 23-39. https://doi.org/10.14786/flr.v7i2.407

Schwartz, S. T., Siegel, A. L., \& Castel, A. D. (2020). Strategic encoding and enhanced memory for positive value-location associations. Memory \& Cognition, 1-17. https://doi.org/10.3758/s13421-02001034-4. Advance online publication.

Shigemune, Y., Tsukiura, T., Kambara, T., \& Kawashima, R. (2014). Remembering with gains and losses: Effects of monetary reward and punishment on successful encoding activation of source memories. Cerebral Cortex, 24(5), 1319-1331. https://doi.org/10.1093/ cercor/bhs415

Siegel, A. L., \& Castel, A. D. (2018a). Memory for important itemlocation associations in younger and older adults. Psychology and Aging, 33(1), 30-45. https://doi.org/10.1037/pag0000209

Siegel, A. L., \& Castel, A. D. (2018b). The role of attention in remembering important item-location associations. Memory \& Cognition, 46(8), 1248-1262. https://doi.org/10.3758/s13421-018-0834-4
Singmann, H., \& Kellen, D. (2019). An introduction to mixed models for experimental psychology. In D. H. Spieler \& E. Schumacher (Eds.), New methods in cognitive psychology (pp. 4-31). Hove: Psychology Press.

Snodgrass, J. G., \& Corwin, J. (1988). Pragmatics of measuring recognition memory: Applications to dementia and amnesia. Journal of Experimental Psychology: General, 117(1), 34-50. https://doi.org/ 10.1037/0096-3445.117.1.34

Spaniol, J., Schain, C., Bowen, H. J. (2014). Reward-enhanced memory in younger and older adults. The Journals of Gerontology, Series B: Psychological Sciences and Social Sciences, 69(5), 730-740. https://doi.org/10.1093/geronb/gbt044

Spencer, W. D., \& Raz, N. (1995). Differential effects of aging on memory for content and context: A meta-analysis. Psychology and Aging, 10(4), 527-539. https://doi.org/10.1037/0882-7974.10.4.527

Stefanidi, A., Ellis, D. M., \& Brewer, G. A. (2018). Free recall dynamics in value-directed remembering. Journal of Memory and Language, 100, 18-31. https://doi.org/10.1016/j.jml.2017.11.004

Wenzel, K., \& Reinhard, M.-A. (2019). Relatively unintelligent individuals do not benefit from intentionally hindered learning: The role of desirable difficulties. Intelligence, 77, Article 101405. https://doi. org/10.1016/j.intell.2019.101405

Wittmann, B. C., Schott, B. H., Guderian, S., Frey, J. U., Heinze, H. J., \& Düzel, E. (2005). Reward-related FMRI activation of dopaminergic midbrain is associated with enhanced hippocampus-dependent longterm memory formation. Neuron, 45(3), 459-467. https://doi.org/ 10.1016/j.neuron.2005.01.010

Publisher's note Springer Nature remains neutral with regard to jurisdictional claims in published maps and institutional affiliations. 